\title{
Active Tissue Stiffness Modulation Controls Valve Interstitial Cell Phenotype and Osteogenic Potential in 3D Culture
}

Authors:

Bin Duan ${ }^{1}$, Ziying Yin $^{2}$, Laura A. Hockaday ${ }^{1}$, Richard L. Magin ${ }^{2}$, Jonathan T. Butcher ${ }^{1 *}$ Affiliation:

${ }^{1}$ Department of Biomedical Engineering, Cornell University, Ithaca, NY

${ }^{2}$ Department of Bioengineering, University of Illinois at Chicago, Chicago, IL

*Corresponding author

Department of Biomedical Engineering

Cornell University, Ithaca, NY, USA.

E-mail address: jtb47@cornell.edu

Phone: (607) 255-3575

Keywords:

Calcific aortic valve disease, stiffness, magnetic resonance imaging, elastography, hydrogel, myofibroblast 


\begin{abstract}
Calcific aortic valve disease (CAVD) progression is a highly dynamic process whereby normally fibroblastic valve interstitial cells (VIC) undergo osteogenic differentiation, maladaptive extracellular matrix (ECM) composition, structural remodeling, and tissue matrix stiffening. However, how VIC with different phenotypes dynamically affect matrix properties and how the altered matrix further affects VIC phenotypes in response to physiological and pathological conditions have not yet been determined. In this study, we develop 3D hydrogels with tunable matrix stiffness to investigate the dynamic interplay between VIC phenotypes and matrix biomechanics. We find that VIC populated within hydrogels with valve leaflet like stiffness differentiate towards myofibroblasts in osteogenic media, but surprisingly undergo osteogenic differentiation when cultured within lower initial stiffness hydrogels. VIC differentiation progressively stiffens the hydrogel microenvironment, which further upregulates both early and late osteogenic markers. These findings identify a dynamic positive feedback loop that governs acceleration of VIC calcification. Temporal stiffening of pathologically lower stiffness matrix back to normal level, or blocking the mechanosensitive RhoA/ROCK signaling pathway, delays the osteogenic differentiation process. Therefore, direct ECM biomechanical modulation can affect VIC phenotypes towards and against osteogenic differentiation in 3D culture. These findings highlight the importance of the homeostatic maintenance of matrix stiffness to restrict pathological VIC differentiation.
\end{abstract}




\section{Introduction}

Calcific aortic valve disease (CAVD) is the third leading cause of cardiovascular disease, resulting in more than 23,000 deaths annually in the United States [1]. This progressively stenotic and sclerotic disease results in ventricular outflow obstruction, elevated blood pressure, and increased stress imparted to the myocardial wall [2]. Features of CAVD include fibrotic thickening of the valve leaflets, oxidized low-density lipoprotein deposition, inflammation, neovascularization, calcified matrix deposition, and bone-like nodule formation [3-5]. Calcific degeneration is mediated in part by valve interstitial cells (VIC), which exhibit a heterogeneous but largely quiescent fibroblastic phenotype in healthy adult valve leaflets $[6,7]$. VIC can become activated and differentiate into a myofibroblastic phenotype in response to microenvironmental cues [8, 9]. Prolonged activation of VIC results in disruption of the highly stratified and biomechanically efficient extracellular matrix (ECM) of the leaflet [10]. The thickened and disorganized ECM in turn further activates VIC and promotes their pathological differentiation towards osteoblast-like phenotypes, which calcify and eventually mineralize this matrix [11]. These dynamic VIC-ECM interactions are thus important components in regulating VIC functions and valve calcification progression, but very little is known about how initial biomechanical properties of matrix potentiate the feedback between VIC differentiation and matrix remodeling [12].

VIC are able to sense the local mechanical properties of ECM through several signaling pathways, like RhoA/ROCK and PI3K/AKT pathway [13-16], and their cellular response to microenvironmental cues are dependent on matrix stiffness [17, 18]. Extensive studies have shown that stiffer substrates $(>15 \mathrm{kPa})$ significantly promoted the 
differentiation of VIC to myofibroblasts under two dimensional (2D) culture [15, 19]. Micro-topographies that induce VIC alignment and elongation on low modulus substrates $(\sim 3 \mathrm{kPa})$ can also stimulate VIC activation [20]. The same group also demonstrated that in situ decreasing the matrix modulus in 2D VIC culture induced cell de-activation [15, 21]. These studies implemented 2D matrix and the matrices were dynamically softened (from pathologically high stiffness to physiological level), which is not physiological process during CAVD progression. In addition, few studies have reported on the role of matrix mechanics in regulating calcification and osteogenic differentiation of VIC. Yip et al. demonstrated that porcine VIC seeded on the surface of collagen gels with lower stiffness, rather than higher stiffness, formed calcified aggregates of viable cells that expressed osteoblast-related transcripts and proteins [22]. However, how altered initial phenotype of VIC affect matrix properties and how the altered matrix further affects VIC phenotype have yet to be determined. Such dynamic interaction between VIC behavior and matrix properties are also hardly investigated in 2D culture. Hydrogel-based 3D culture platforms provide an in vitro tissue-like microenvironment that mimics ECM and regulates VIC behaviors both physiologically and pathologically [23, 24]. Hydrogel culture platforms can also be dynamically remodeled by resident cells, thereby enabling investigation of microenvironmental feedback on cellular differentiation.

In the current study, we quantitatively analyzed the interplay between human aortic VIC (HAVIC) differentiation and matrix biomechanical remodeling. We implemented a bioactive hydrogel platform with tunable matrix stiffness with encapsulated HAVIC. The HAVIC in healthy, fibrotic, and calcification prone environments were conditioned. We assessed the roles of stiffness on VIC phenotypes and determined how HAVIC phenotype 
affects compaction rate, stiffness and ECM content of constructs. Then the role of RhoA/ROCK signaling (activation or inhibition) in HAVIC osteogenic differentiation was evaluated for these ECM conditions. Finally, we show that temporally modulating hydrogel stiffness in live constructs can retard the VIC calcification process.

\section{Materials and Methods}

\subsection{Cell isolation and cell culture}

HAVIC were isolated from the aortic valve leaflets of the donor heart from a 12-year old patient undergoing cardiac transplant for deficient myocardial contractility [25]. The valve leaflets were functionally normal on echo, and upon excision were found not to contain any calcific deposits or thickened lesions. Tissue was procured with consent as approved by the Institutional Review Board of Weill-Cornell Medical College in New York City. VIC were cultured in MCDB131 medium (Sigma) supplemented with 10\% fetal bovine serum (FBS; Invitrogen), $1 \%$ penicillin/streptomycin (P/S; Invitrogen), 0.25 $\mu \mathrm{g} / \mathrm{L}$ recombinant human fibroblast growth factor basic (rhFGF-2; Invitrogen) and $5 \mu \mathrm{g} / \mathrm{L}$ recombinant human epidermal growth factor (rhEGF; Invitrogen). Multiple cell batches were used from passages 4-8 [26].

\subsection{Polymer modification, hydrogel preparation and conditioning}

Photocrosslinkable hyaluronic acid (HA, Novozymes, $1200 \mathrm{kDa}$ ) and gelatin (Gel, from bovine skin, Sigma) were synthesized as previously reported [27] through the reaction of methacrylic anhydride (Sigma) with $0.5 \%$ HA or $10 \%$ Gel in deionized water. The degree of methacrylation was approximately estimated to be $22.5 \%$ for Me-HA and $61.1 \%$ for Me-Gel based on the ${ }^{1} \mathrm{H}$ NMR spectroscopy [25]. 
For homogeneous hydrogel preparation, methacrylated HA (Me-HA, 0.5\%w/v) and methacrylated Gel (Me-Gel, 1\%w/v) were dissolved in cell culture medium with or without cell suspension $\left(4 \times 10^{6} \quad\right.$ cells $\left./ \mathrm{ml}\right)$ and $0.05 \% \quad \mathrm{w} / \mathrm{v}$ 2-hydroxy-1(4-(hydroxyethox)pheny)-2-methyl-1-propanone (Irgacure 2959; CIBA Chemicals). The gel precursor was transferred into silicone molds $(8 \mathrm{~mm}$ in diameter, 1 mm thickness) and subsequently exposed to $365 \mathrm{~nm}$ UV light (EN-280L, Spectroline, 2.0 $\mathrm{mW} / \mathrm{cm}^{2}$ ) for $1 \mathrm{~min}$ or $5 \mathrm{~min}$ (Fig. 1A).

The cell-hydrogel constructs were maintained in control medium (MCDB medium with $10 \% \mathrm{FBS}, 1 \% \mathrm{P} / \mathrm{S}$, rhFGF-2 and EGF) for $24 \mathrm{~h}$ and then cultured in control medium, activation medium (MCDB medium with $10 \% \mathrm{FBS}, 1 \% \mathrm{P} / \mathrm{S}$ and $5 \mathrm{ng} / \mathrm{ml}$ transforming growth factor beta 1 (TGF $\beta 1$, PeproTech)), or osteogenic medium (MCDB medium with $10 \%$ FBS, $1 \% \mathrm{P} / \mathrm{S}, 5 \mathrm{ng} / \mathrm{ml}$ TGF $\beta 1,100 \mathrm{nM}$ dexamethasone (Sigma), $10 \mathrm{mM}$ $\beta$-glycerophosphate (Sigma) and $50 \mu \mathrm{M}$ ascorbic acid (Sigma)) up to 21 days.

Heterogeneous photocrosslinking of cell-laden hydrogels were fabricated by exposing portions of the same hydrogel to UV light for different time points (Fig. 1B). The cell-hydrogel precursor solution was first loaded in a square silicone mold $(15 \mathrm{~mm} \times$ $15 \mathrm{~mm}, 1 \mathrm{~mm}$ thickness) and photocrosslinked for $1 \mathrm{~min}$. Next we used a black photomask (Fineline Imaging) to cover part of the hydrogels to block the UV light and then the constructs were further exposed to UV light for another $4 \mathrm{~min}$. Thus, part of the construct was photocrosslinked for $1 \mathrm{~min}$ and the other part was photocrosslinked for 5 min. The cell-hydrogel constructs with heterogeneous photocrosslinking pattern were conditioned in control medium for 1 day and osteogenic media for another 20 days. 
For in situ stiffening of HAVIC laden hydrogels, the constructs with 1 min photocrosslink were cultured in osteogenic media for 7 days and then were stiffened via radical polymerization by UV exposure for another 4 min (Fig. 1C). Before hydrogel stiffening, the constructs were placed in a 6-well plate with Irgacure 2959 containing media $(0.05 \mathrm{wt} \%)$ and incubated for $30 \mathrm{~min}$ at $37{ }^{\circ} \mathrm{C}$, followed by exposure to $\mathrm{UV}$. Immediately after exposure, the constructs were washed three times with fresh media and conditioned in osteogenic media for another 14 days. Live/dead assay showed that the cell viability did not significantly decrease during the stiffening process (Fig. S1).

For pharmacological activation or inhibition of Rho kinase (ROCK), ROCK activator oleoyl-L-a-lysophosphatidic acid sodium salt (20 $\mu \mathrm{M}$ LPA, Sigma) or ROCK inhibitor Y27632 (10 $\mu$ M, Sant Cruz) was added to the osteogenic media.

\subsection{Dynamic mechanical analysis (DMA) and microscopic magnetic resonance} elastography $(\mu M R E)$

Uniaxial compressive test of different hydrogels with (at day 1, 7 and 21) or without cells were performed using a dynamic mechanical analyzer (TA Instruments, DMA Q800) at a loading speed of $0.50 \mathrm{~N} / \mathrm{min}$. The bulk compressive moduli were calculated from the slope of the initial linear region (10\%-15\% strain for compressive test) of the respective stress-strain curves.

$\mu \mathrm{MRE}$ experiments were performed on a horizontal bore Agilent 9.4 Tesla MR Scanner (310/ASR, Agilent Technologies, Santa Clara, CA). A linear triple-axis gradient system with a maximum magnetic field gradient strength of $1000 \mathrm{mT} / \mathrm{m}$ and a $39 \mathrm{~mm}$ diameter quadrature radiofrequency coil were used [28, 29]. The hydrogel samples were embedded in a mixture gel of $0.4 \%(w / v)$ agarose (SeaKem LE Agarose, Lonza, 
Rockland, ME) and 1\% (w/v) gelatin (food grade, Knox) in a customized MRE cylindrical plastic container (height $=40 \mathrm{~mm}$; inner diameter $=30 \mathrm{~mm}$ ). The container was attached to a piezo-ceramic actuator, which was excited with an amplified sinusoidal waveform of frequency $400 \mathrm{~Hz}$. The transverse vibratory motion was then transmitted to the sample. The agarose/gelatin mixture gel was used to promote the transfer of shear wave motions into the enclosed samples. Shear motions generated within the sample were encoded into shear wave images using a gradient-echo based MRE pulse sequence. From the wave image data, the magnitude of the complex shear stiffness (an approximation of the magnitude of the complex shear modulus) was estimated using the local frequency estimation inversion algorithm with directional filtering [28].

\subsection{Measurement of gel compaction}

Representative images of hydrogel samples $(n=6)$ were taken using a Zeiss Discovery v20 stereo microscope (Spectra Services, Inc.). In order to qualitatively demonstrate gel compaction throughout the time course in different culture conditions, the areas of the hydrogels at selected time points were measured and compared with their original sizes at day 0 .

\subsection{ECM components analysis}

Dimethylmethylene blue (DMMB) assay was performed to measure the sulfated glycosaminoglycan (GAG) production in the hydrogels after 7 and 21 day culture [25]. The constructs $(\mathrm{n}=5)$ were digested with $300 \mu \mathrm{g} / \mathrm{ml}$ papain in $50 \mathrm{mM}$ phosphate buffer (pH 6.5), containing $5 \mathrm{mM}$ cysteine and $5 \mathrm{mM}$ EDTA for $16 \mathrm{~h}$ at $60{ }^{\circ} \mathrm{C}$. GAG concentration was calculated by calibrating against a standard curve obtained with shark chondroitin sulfate (Sigma). The total collagen content was determined using the 
hydroxyproline assay [30, 31]. The samples were hydrolyzed by $4.8 \mathrm{~N} \mathrm{HCl}$ and then the dried hydrolyzates were then treated with chloramine $\mathrm{T}$ reagent for oxidation. The Ehrlich's aldehyde reagent was reacted with samples at $65{ }^{\circ} \mathrm{C}$ for $20 \mathrm{~min}$ in order to generate chromospheres. The amount of hydroxyproline was measured with the microplate reader at $550 \mathrm{~nm}(\mathrm{n}=5)$. The total elastin content in the cell-laden hydrogels $(n=4-6)$ were measured using the colorimetric Fastin elastin assay kit (Biocolor Ltd, UK), following the manufacturer's instructions. Results for GAG, collagen and elastin contents were normalized using the wet weight of the constructs and expressed in units of $\mu \mathrm{g} / \mathrm{g}$.

\subsection{Alkaline phosphatase (ALP) staining and activity}

ALP staining was performed after 28 days in culture by using the alkaline phosphatase leukocyte kit (Sigma) according to the manufacturer's protocol. The ALP activity of encapsulated cells was also measured. Briefly, the cell laden hydrogels were lysed with lysis buffer and $50 \mu \mathrm{l}$ lysate mixed with $200 \mu \mathrm{l}$ ALP substrate solution containing p-nitrophenyl phosphate (pNPP) (Sigma) at $37{ }^{\circ} \mathrm{C}$ for $10 \mathrm{~min}$. The reaction was stopped by the addition of $50 \mu \mathrm{l}$ of $3 \mathrm{~N} \mathrm{NaOH}$ and then the production of $p$-nitrophenol in the presence of ALPase was measured by monitoring the absorbance of the solution at a wavelength of $405 \mathrm{~nm}$ using a microplate reader (Bio-Tek Instruments). The total protein content was determined using BCA assay kit (Pierce, Rockford, IL, USA) with bovine serum albumin as a standard and the ALP activity was expressed as $\mu$ mol of $p$-nitrophenol formation per minute per milligram of total proteins $(\mu \mathrm{mol} / \mathrm{min} / \mathrm{mg}$ protein).

\subsection{Immunohistochemistry staining}


For immunofluorescent staining, cell-laden hydrogels were fixed in $4 \%$ paraformaldehyde, permeabilized in $0.2 \%$ Triton $\mathrm{X}-100$ and then blocked with $1 \%$ bovine serum albumin (BSA) overnight at $4{ }^{\circ} \mathrm{C}$. Hydrogels were treated with primary antibodies to vimentin (1:100, mouse monoclonal anti-vimentin, Invitrogen) and monoclonal anti- $\alpha$-smooth muscle actin ( $\alpha$ SMA)-Cy3 antibody (1:200, Sigma), or Runx2 (1:100, mouse monoclonal anti-Runx2, Sigma), osteocalcin (OCN, 1:100, mouse monoclonal anti-osteocalcin, Abcam) overnight at $4{ }^{\circ} \mathrm{C}$. Secondary fluorescent antibodies were incubated for $2 \mathrm{~h}$ and nuclear counterstaining (via Draq 5, 1: 1000, Biostatus) were performed for 30 minutes at room temperature. The stained samples were imaged with Zeiss 710 CLSM.

\subsection{RNA isolation and quantitative real time polymerase chain reaction (PCR)}

Total RNA was extracted from cell-laden hydrogels using QIA-Shredder and RNeasy mini-kits (QIAgen) according to the manufactures' instructions. Thirty nanograms of total RNA was synthesized into first strand cDNA in a $20 \mu \mathrm{L}$ reaction using iScript cDNA synthesis kit (BioRad Laboratories). Real-time PCR analysis was performed in a CFX Connect Real-Time PCR detection system (Bio-Rad) using SsoAdvanced SYBR Green Supermix (Bio-Rad). cDNA samples were analyzed for the gene of interest and for the housekeeping gene $18 \mathrm{~S}$ rRNA. The level of expression of each target gene was calculated using comparative $\mathrm{Ct}\left(2^{-\Delta \Delta \mathrm{Ct}}\right)$ method.

\subsection{Western blot}

For western blotting, the cell-hydrogel constructs were washed twice with ice-cold PBS, flash frozen in liquid nitrogen and lysed in hot laemmli buffer. The lysates were sonicated and microcentrifuged, and the protein concentrations were determined by Pierce $660 \mathrm{~nm}$ 
Protein Assay. The lysates were loaded into a 4-15\% gradient precast gel (Bio-Rad, Hercules, CA) and transferred to nitrocellulose membrane (Thermo Scientific, Rockford, IL). The membranes were blocked in Odyssey Blocking Buffer (Li-Cor) and incubated in mouse monoclonal anti-osteocalcin (1:1000, Abcam), rabbit anti-RhoA antibody (1:1000, Cell Signaling Technology) and mouse anti-human GAPDH (1:10,000, Invitrogen), respectively. Blots were imaged using the Odyssey Infrared system (Li-Cor) after incubation with secondary antibodies.

\subsection{Statistical analysis}

All quantitative data are expressed as the mean \pm standard deviation (SD). Statistical analysis was performed using ANOVA with Scheffé post-hoc tests. A value of $p<0.05$ was considered to be statistically significant.

\section{Results}

\subsection{Dynamic changes of mechanical properties of HAVIC laden hybrid hydrogels}

Bulk compressive properties of acellular hydrogels were measured by DMA (Fig. 2A). As expected, the modulus correlated with the photocrosslink time. With increasing the photocrosslink time from $1 \mathrm{~min}$ to $5 \mathrm{~min}$, the stiffness of hybrid hydrogels significantly increased from $1.33 \pm 0.17 \mathrm{kPa}$ to $3.33 \pm 0.19 \mathrm{kPa}(p<0.01)$. The $\mu \mathrm{MRE}-$ derived shear stiffness, which mimics the shear modulus, was measured by embedding hydrogel samples in an agarose/gelatin mixture. In gels and biological tissues, the shear modulus is typically $1 / 3$ of the Young's modulus [32]. A representative shear wave image is displayed in Fig. 2B. The computed shear modulus maps were computed from this data for $1 \mathrm{~min}$ and $5 \mathrm{~min}$ photocrosslink hydrogels using the LFE algorithm (Fig. 2B). The shear modulus was determined to be $0.33 \pm 0.02 \mathrm{kPa}$ for 1 min hydrogel, and $1.01 \pm 0.05$ 
$\mathrm{kPa}$ for 5 min hydrogel, which correspond well with the compressive modulus values, and confirmed that the stiffness also increased with increasing photocrosslink time, probably due to the increase of crosslinking density. Hereafter, hydrogel with $1 \mathrm{~min}$ photocrosslink and initial acellular stiffness of $1 \mathrm{kPa}$ is denoted as $1 \mathrm{kPa}$ hydrogel, while hydrogel with 5 min photocrosslink and initial acellular stiffness of $3 \mathrm{kPa}$ is $3 \mathrm{kPa}$ hydrogel.

The dynamic changes of compressive modulus of cell-laden hydrogels at day 1, day 7 and day 21 were measured by DMA. For $3 \mathrm{kPa}$ hydrogels, the stiffness of HAVIC-laden hydrogels at day 1 decreased relative to acellular baseline (Fig. 2D). This is likely due to the presence of in situ encapsulated cells that create material voids at this early time rather than bearing the load. With increasing the culture time, tissue stiffness increased to the acellular level for all treatment conditions. 3D cultured VIC in control media maintained tissue stiffness to day 21 , while the VIC conditioned in activation or osteogenic media markedly enhanced tissue stiffness, especially in the osteogenic media. For $1 \mathrm{kPa}$ hydrogel, the stiffness was comparable to their respective acellular controls at day 1 (Fig. 2E). The early maintenance of hydrogel stiffness is probably because the softer hydrogels have much lower crosslink density and larger mesh size. With increasing culture time, only the VIC in $1 \mathrm{kPa}$ hydrogels conditioned in the osteogenic media increased stiffness, while the other conditions maintained the stiffness at the same level. Interestingly, the stiffness of $1 \mathrm{kPa}$ constructs increased much greater $(1.05 \pm 0.23 \mathrm{kPa}$ to $5.02 \pm 0.56 \mathrm{kPa}$ Day 7 vs. $21, p<0.05)$ during conditioning in osteogenic media when compared to $3 \mathrm{kPa}$ hydrogels $(3.87 \pm 1.38 \mathrm{kPa}$ to $6.96 \pm 1.09 \mathrm{kPa}$, day 7 vs. $21, p<0.05)$. 
The shear stiffness of hydrogel samples with different photocrosslink time determined by $\mu \mathrm{MRE}$ showed similar trends. With increasing the culture time, the hydrogels conditioned in the activation media and osteogenic media had significantly increased shear stiffness (Fig. S2).

\subsection{Hydrogel compaction}

Representative images of hydrogels in different conditions at day 0 (immediately after gelation) and at day 21 are shown in Fig. S3. At the initial time point (day 0), the $1 \mathrm{kPa}$ hydrogels had a larger area than $3 \mathrm{kPa}$ hydrogels due to a higher swell ratio. All the hydrogels compacted with increasing culture time. Constructs compacted depending on initial stiffness (photocrosslink time) and culture conditions. Generally, the compaction rate of $1 \mathrm{kPa}$ VIC-laden hydrogels was significantly higher than that of $3 \mathrm{kPa}$ VIC-laden hydrogels under the same culture condition. The HAVIC populated hydrogels conditioned in osteogenic media compacted significantly more than those in activation media, while the constructs in control media had the lowest compaction at day 21 (Fig. S3).

\subsection{Dynamic changes of ECM contents}

The level of GAG, collagen and elastin within HAVIC populated hydrogels were compared at 7 and 21 days of culture. Generally, GAG, collagen and elastin content significantly increased with increasing culture time (Fig. 3). HAVIC populated hydrogels with higher initial stiffness contained more GAG, collagen and elastin than $1 \mathrm{kPa}$ hydrogels when conditioned in activation media after 7 day and 21 days of culture. In

contrast, GAG, collagen, and elastin contents were greatly higher in $1 \mathrm{kPa}$ hydrogels than $3 \mathrm{kPa}$ hydrogels when conditioned in osteogenic media at day 21. In control media at day 
7 and day 21, $1 \mathrm{kPa}$ hydrogels showed the same level of GAG content as $3 \mathrm{kPa}$ hydrogels (Fig. 3A), whereas collagen and elastin content in $3 \mathrm{kPa}$ hydrogels were much higher than those in the $1 \mathrm{kPa}$ hydrogels (Fig. 3B and C). In the control media, all the constructs had increased total ECM content (collagen+GaG+elastin) without affecting the stiffness (Fig. 3D). This indicates a balance between material degradation and ECM deposition. When conditioned in osteogenic media, ECM content increased with increasing construct stiffness in the same type of hydrogels during culture, suggesting that the newly secreted ECM stiffened the hydrogels. The $1 \mathrm{kPa}$ hydrogels in osteogenic media had the highest total GAG, collagen and elastin content after 21 day conditioning (Fig. 3D and Fig. S4).

\subsection{HAVIC phenotypes in response to hydrogel stiffness and culture conditioning}

The encapsulated HAVIC expressed both $\alpha$ SMA and vimentin within all the hydrogel samples after 21-day culture, as shown in Fig. 4A. As expected, HAVIC conditioned in activation or osteogenic media mostly exhibited myofibroblastic-like differentiation indicated by positive $\alpha$ SMA. Only VIC conditioned in osteogenic media showed detectable Runx2, which was extensively nuclear expressed in the HAVIC encapsulated in $1 \mathrm{kPa}$ hydrogels, indicating it is transcriptionally active. In addition, only HAVIC populated in $1 \mathrm{kPa}$ hydrogels in osteogenic media showed positive osteocalcin (OCN) expression (Fig. 4B).

We next studied the dynamic relationship between the matrix stiffness and the gene expression of vimentin, $\alpha \mathrm{SMA}$, Runx2 and OCN (Fig. 4C). With increasing matrix stiffness for $1 \mathrm{kPa}$ and $3 \mathrm{kPa}$ hydrogel during culture, the expression of vimentin was downregulated and the expression of $\alpha \mathrm{SMA}$ was upregulated in active and osteogenic media. Similarly, the expressions of Runx2 and OCN were all upregulated with 
increasing matrix stiffness. Of note, HAVIC encapsulated in $3 \mathrm{kPa}$ hydrogels in osteogenic media showed significantly decreased expression of vimentin and increased expression of aSMA, whereas HAVIC in $1 \mathrm{kPa}$ hydrogels in osteogenic media significantly upregulated Runx2 and OCN. These results indicate that osteogenic media promoted myofibroblastic differentiation in the stiffer $3 \mathrm{kPa}$ VIC populated hydrogels, but promoted osteogenic differentiation in less stiff $1 \mathrm{kPa}$ VIC populated hydrogels.

\subsection{Dynamic changes of ALP activity}

Only $1 \mathrm{kPa}$ VIC hydrogels conditioned in osteogenic media expressed positive ALP at day 7, while the constructs in other conditions were negative to ALP staining (Fig. 5A). After 21-day culture, HAVIC in activation media expressed very limited ALP and only osteogenic media promoted the ALP expression. We also observed that the ALP activity significantly increased with increasing the local microenvironment stiffness (Fig. 5B). Importantly, osteogenic differentiation of HAVIC occurred only when the constructs were exposed to calcification prone media, regardless of initial matrix stiffness. This indicates that matrix stiffness alone (at least within this range) is insufficient to induce osteogenic differentiation, but rather works together with soluble factors to regulate HAVIC differentiation and calcification [22].

3.6 Verification of osteogenic differentiation capacity difference in heterogeneous hydrogel constructs

In order to verify the osteogenic differentiation capacity difference in hydrogels with different initial stiffness, we generated heterogeneous hydrogel constructs by exposing the HAVIC-laden hydrogels to UV light for different time in the same construct (Fig. 1B). As before, HAVIC populated in $1 \mathrm{kPa}$ hydrogel expressed more ALP when compared to 
$3 \mathrm{kPa}$ hydrogel counterpart, showing extensive and concentrated red color (Fig. 6A). The boundary between $1 \mathrm{kPa}$ and $3 \mathrm{kPa}$ photocrosslink hydrogel was clearly identifiable, but not a straight line because of differential hydrogel contraction and remodeling. Cell migration from $1 \mathrm{kPa}$ hydrogel to $3 \mathrm{kPa}$ hydrogel was also observed. HAVIC in $1 \mathrm{kPa}$ hydrogels expressed significantly higher ALP gene expression than those in $3 \mathrm{kPa}$ hydrogels $(p<0.01$, Fig. 6B).

3.7 In situ hydrogel stiffening inhibited/delayed the osteogenic differentiation of HAVIC

To test whether ECM stiffness mediated VIC differentiation could be modified dynamically, we temporally stiffened $1 \mathrm{kPa}$ hydrogels after 7 days of culture using the same photoinitiator/UV light method as before. In situ stiffened hydrogels showed lower compaction and a higher modulus than the $1 \mathrm{kPa}$ hydrogels, comparable to the $3 \mathrm{kPa}$ hydrogels (Fig. 7A and B) after additional 14 days culture. The temporal stiffening process also significantly decreased the ALP activity (Fig. 7C), ALP, and OCN expression (Fig. 7D) of HAVIC compared to HAVIC populated in $1 \mathrm{kPa}$ hydrogels. These results support the hypothesis that in situ stiffening of pathologically compliant matrix can delay osteogenic differentiation and matrix calcification.

\subsection{The role of RhoA/ROCK pathway on osteogenic differentiation of HAVIC}

The RhoA/ROCK pathway regulates contractility and stress fiber formation and has been previously showed to regulate heart valve calcification $[16,33]$. We examined the role of RhoA/ROCK pathway in the pro-osteogenic differentiation of HAVIC in our 3D hybrid hydrogels by treating the $1 \mathrm{kPa}$ constructs with ROCK inhibitor (Y-27632) and activator (LPA). The addition of ROCK inhibitor downregulated the expression of vimentin, Runx2, and OCN (Fig. 8A). Interestingly, when ROCK activity was stimulated by 
addition of LPA, the expressions of aSMA, Runx2, and OCN were maintained at the same level of untreated group. But the treatment of ROCK activator significantly decreased the expression of vimentin. Western blot results shown in Fig. 8B confirmed that the addition of ROCK inhibitor decreased the relative amount of RhoA expression normalized to GAPDH from $\sim 0.58$ to $\sim 0.17$ fold, and OCN expression from $\sim 0.20$ to $\sim 0.06$ fold for HAVIC. These results indicate that the effects of RhoA/ROCK activity on VIC phenotypes are regulated by the stiffness of the ECM.

\section{Discussion}

CAVD progression is a highly dynamic process involving pathological VIC activation and osteogenic differentiation, maladaptive structural remodeling, and leaflet matrix stiffening [10]. These events create a permissive microenvironment in which osteogenic transformation occurs, leading to development of CAVD [12]. Current 2D and 3D culture models (culture plates or scaffolds/hydrogels) mainly focus on how matrix stiffness affects VIC phenotypes without considering how VIC remodel matrix and how these altered matrix properties further affect VIC phenotypes. Recently, Sider et al found that early valve lesions contained proteoglycan-rich deposits in the fibrosa layer, which could impair local mechanical stiffness of fibrosa layer [34]. Mabry et al. also reported that dynamically stiffened hydrogels (from $0.24 \mathrm{kPa}$ to 4 and $12 \mathrm{kPa}$ ) reduced activation of encapsulated VIC [35]. There is therefore a significant need to develop a more physiologically relevant culture platform for replicating the interplay between VIC and ECM in the progression of CAVD so as to identify functional mechanisms and to develop effective diagnostic and therapeutic treatments. In addition, much has been learned about porcine valve cell phenotype in response to static culture conditions, far less is known 
about the dynamics of human valve cell phenotype responses to changing 3D culture environments. In this study, we identify a strong feedback between initial matrix stiffness and HAVIC differentiation/matrix remodeling. We developed 3D hydrogels with tunable matrix stiffness to investigate the dynamic interplay between HAVIC phenotype and matrix biomechanical remodeling in response to physiological, activation and calcification prone environments. The hydrogels consisted of Me-HA and Me-Gel and the mechanical properties were tuned by varying the photocrosslink time from 1 min to 5 min. The compressive modulus of acellular hydrogels with 5 min photocrosslink was tuned to match that of the ventricularis $(\sim 3 \mathrm{kPa})$ and fibrosa $(\sim 5 \mathrm{kPa})$ layers of aortic heart valve leaflet measured by micropipette aspiration [36], and the shear stiffness was comparable to that of valve leaflets in physiological condition under $25 \%$ strain $(\sim 1 \mathrm{kPa})$ [37]. The mechanical properties of acellular 1 min photocrosslink hydrogels $(\sim 1 \mathrm{kPa})$ were significantly lower than those of 5 min hydrogels $(\sim 3 \mathrm{kPa})$ and native valve leaflets.

This study demonstrates that the stiffness of constructs dynamically changed in response to different medium conditions and the altered stiffness further regulated HAVIC phenotypes. Under physiological media conditions, the stiffness of HAVIC populated hydrogel constructs was maintained by balancing hydrogel degradation and ECM remodeling. Both material degradation and matrix secretion significantly affect the hydrogel properties and this is a very dynamic process. The balance of this process determines the overall stiffness of the constructs. Both modification of crosslink density and hydrolysis of biomolecules happen during degradation process, but again by multiple mechanisms. The constructs stiffened under pathological conditions by enhanced hydrogel compaction and matrix deposition, but the stiffening effect was dependent on 
initial matrix stiffness. Previous studies demonstrate that hydrogel stiffness for fibroblast-myofibroblast transition of VIC in 3D culture is lower than that presented in 2D culture (4.80-9.60 $\mathrm{kPa}$ [19] or $\sim 7 \mathrm{kPa}$ [21]). Our current results find that stiffened constructs with initially valve leaflet layers-like stiffness significantly promote myofibroblastic phenotype of HAVIC. This confirms that the component of the TGF $\beta$ pathway is regulated in a stiffness-dependent manner. Meyer-ter-Vehn et al. reported that TGF $\beta 1$ induced myofibroblast transdifferention of human tendon fibroblasts was attenuated on compliant matrix in 2D culture by decreasing p38 activation [38]. Wipff et al. also observed a modest matrix stiffness $(\sim 5 \mathrm{kPa})$ dependent increase in fibroblast activation of TGF $\beta 1$ [39]. Although TGF $\beta 1$ is present in human calcified aortic valve leaflets [40], exogenous TGF $\beta 1$ alone does not provoke any HAVIC osteogenic differentiation, which suggests that TGF $\beta 1$ signaling is associated with myofibroblast differentiation in CAVD, but probably not osteogenic processes.

In contrast to constructs with initially valve leaflet layers-like stiffness (5 min photocrosslink, $\sim 3 \mathrm{kPa}$ ), HAVIC populated hydrogels with lower initial stiffness (1 min photocrosslink, $\sim 1 \mathrm{kPa}$ ) facilitated osteogenic differentiation of HAVIC, showing much higher ALP activity and OCN expression. Interestingly, these results are in contrast with several recent 3D studies which described that the osteogenic commitment of MSC favored the 3D hydrogels with stiffer elastic modulus [41-43]. In these studies, however, the encapsulated cells maintained a grossly rounded morphology due to low adhesivity or degradability or high stiffness. The degree of cytoskeletal tension, adhesion ligand density and cell density also directly correlate with the matrix modulus and regulate MSC differentiation [44, 45]. In this work, we find that well spread HAVIC within low 
stiffness hydrogels allows them to apply tensions, resulting in more compaction and higher cell density, which initiate osteogenic differentiation. Similar observations were also reported by other studies [44, 46]. The initiated osteogenic differentiation significantly increased construct stiffness and the stiffened constructs further upregulated both early and late osteogenic markers. Therefore, this in vitro culture system replicates what is known about the dynamic feedback loop in early stages of valve calcification.

Pro-calcification of HAVIC is related to a tension-mediated switch involving the RhoA/ROCK signaling pathways. Hampering the contractile apparatus of HAVIC by ROCK inhibitors (Y27632) inhibits the osteogenic differentiation. Similarly, Gu et al. demonstrated that nodule formation in VIC cultures was associated with RhoA/ROCK activity [47]. Bouchareb et al. also found that RhoA/ROCK mediates the transportation of ectonucleotide pyrophosphatase/phosphodiesterase (ENPP1), an ecto-nucleotidase enzyme, to the plasma membrane, which promotes strain induced mineralization of VIC [16]. However, addition of ROCK activator (LPA) promoted myofibroblastic transdifferentiation of HAVIC, without affecting their osteogenic differentiation. This indicates that apart from RhoA/ROCK signaling pathway, there are many other potential avenues that may be stimulating VIC differentiation and calcification. For example, MAPK/ERK signaling is significantly elevated in the nodule-forming environments [48] and the Wnt/ $\beta$-catenin signaling pathway is related to early stage of osteogenic differentiation and calcification of VIC [49].

Very little is known about how ECM mechanical properties change at the early stage of CAVD and how the altered ECM organization, composition and mechanical properties affect CAVD progression. This study provides initial evidence that local ECM disruption 
leading to tissue stiffness decreases from physiological level to pathological level may accelerate CAVD progression and be one of the early mechanisms of CAVD (Fig. 9A). There may be several other potential mechanisms whereby matrix stiffness has different change rates and forms (Fig. 9A). Our current in vitro model replicates the dynamic pathological process of matrix stiffness changes and VIC calcification. Within pathological microenvironment(s), the constructs with stiffness lower than normal level facilitate osteogenic differentiation of HAVIC and the differentiation is mediated by RhoA/ROCK signaling pathway. Temporal stiffening of lower stiffness matrix to valve leaflet layers-like stiffness or blocking RhoA/ROCK signaling pathway activates HAVIC and may delay or partially inhibit osteogenic differentiation process (Fig. 9B). These findings illustrate that ECM mechanical properties are implicated in CAVD progression and highlight the importance of homeostatic maintenance of matrix stiffness to restrict pathological VIC differentiation.

\section{Conclusions}

In the current study, we implement 3D hydrogel systems with tunable stiffness that matches those of healthy and disrupted native valve leaflet layers respectively to study the potential mechanisms of VIC calcification. We identify a strong interplay between matrix stiffness and VIC response to physiological and pathological conditions within 3D hydrogels. The in vitro model replicates the dynamic pathological process of VIC calcification. HAVIC populated hydrogels with lower initial stiffness than valve leaflet layers facilitate osteogenic differentiation, which is mediated by RhoA/ROCK signaling pathway. Our findings provide an improved understanding of VIC-matrix interactions to aid in interpretation of VIC calcification studies in vitro and suggest that ECM disruption 
resulting in local tissue stiffness decreases may promote CAVD progression. Acknowledgments

This work was funded by the American Heart Association Postdoctoral Fellowship (13POST17220071), Morgan Family Foundation, The Hartwell Foundation, the National Science Foundation (CBET-0955172) NSF Graduate Research Fellowship, Foundation LeDucq MITRAL Transnational Network, and the National Institutes of Health (HL110328, HL118672, HL1287845). The MRE work was supported, in part, by the NIBIB Grant (EB007537) to RLM. We thank Dr. Jonathan Chen in the Department of Cardiothoracic Surgery at Weill Cornell Medical College for providing human aortic valve tissue. This work made use of the Cornell Center for Materials Research Facilities supported by the National Science Foundation under Award Number DMR-1120296. We

would like to thank he Cornell University Biotechnology Resource Center (BRC) Imaging Facility for their assistance with CLSM imaging and the facilities are supported by the National Institutes of Health (NIH 1S10RR025502).

\section{Disclosure Statement}

No competing financial interests exist. 


\section{References}

[1] V.L. Roger, A.S. Go, D.M. Lloyd-Jones, E.J. Benjamin, J.D. Berry, W.B. Borden,

D.M. Bravata, S. Dai, E.S. Ford, C.S. Fox, H.J. Fullerton, C. Gillespie, S.M. Hailpern, J.A. Heit, V.J. Howard, B.M. Kissela, S.J. Kittner, D.T. Lackland, J.H. Lichtman, L.D. Lisabeth, D.M. Makuc, G.M. Marcus, A. Marelli, D.B. Matchar, C.S. Moy, D. Mozaffarian, M.E. Mussolino, G. Nichol, N.P. Paynter, E.Z. Soliman, P.D. Sorlie, N. Sotoodehnia, T.N. Turan, S.S. Virani, N.D. Wong, D. Woo, M.B. Turner, A.H.A.S. Comm, S.S. Subcomm, Heart disease and stroke statistics-2012 Update a report from the American Heart Association, Circulation 125 (2012) E2-E220.

[2] W.D. Merryman, Mechano-potential etiologies of aortic valve disease, J. Biomech. 43 (2010) 87-92.

[3] D.A. Towler, Molecular and cellular aspects of calcific aortic valve disease, Circ. Res. 113 (2013) 198-208.

[4] N.M. Rajamannan, R.O. Bonow, S.H. Rahimtoola, Calcific aortic stenosis: an update, Nat. Clin. Pract. Card. 4 (2007) 254-262.

[5] J.D. Miller, R.M. Weiss, D.D. Heistad, Calcific aortic valve stenosis: Methods, models, and mechanisms, Circ. Res. 108 (2011) 1392-1412.

[6] E. Aikawa, P. Whittaker, M. Farber, K. Mendelson, R.F. Padera, M. Aikawa, F.J. Schoen, Human semilunar cardiac valve remodeling by activated cells from fetus to adult - Implications for postnatal adaptation, pathology, and tissue engineering, Circulation 113 (2006) 1344-1352.

[7] D.L. Mulholland, A.I. Gotlieb, Cell biology of valvular interstitial cells, Can. J. Cardiol. 12 (1996) 231-236.

[8] P.A. Taylor, P. Batten, N.J. Brand, P.S. Thomas, M.H. Yacoub, The cardiac valve interstitial cell, Int. J. Biochem. Cell. B 35 (2003) 113-118.

[9] A.C. Liu, A.I. Gotlieb, Transforming growth factor-beta regulates in vitro heart valve repair by activated valve interstitial cells, Am. J. Pathol. 173 (2008) 1275-1285.

[10] J.H. Chen, C.A. Simmons, Cell-matrix interactions in the pathobiology of calcific aortic valve disease critical roles for matricellular, matricrine, and matrix mechanics cues, Circ. Res. 108 (2011) 1510-1524. 
[11] H. Wang, L.A. Leinwand, K.S. Anseth, Cardiac valve cells and their microenvironment-insights from in vitro studies, Nat. Rev. Cardiol. 11 (2014) 715-727. [12] C. Li, S.Y. Xu, A.I. Gotlieb, The progression of calcific aortic valve disease through injury, cell dysfunction, and disruptive biologic and physical force feedback loops, Cardiovasc. Pathol. 22 (2013) 1-8.

[13] J.T. Butcher, C.A. Simmons, J.N. Warnock, Review-Mechanobiology of the aortic heart valve, J. Heart Valve Dis. 17 (2008) 62-73.

[14] K.J. Rodriguez, K.S. Masters, Regulation of valvular interstitial cell calcification by components of the extracellular matrix, J. Biomed. Mater. Res. A 90A (2009) 1043-1053. [15] H. Wang, M.W. Tibbitt, S.J. Langer, L.A. Leinwand, K.S. Anseth, Hydrogels preserve native phenotypes of valvular fibroblasts through an elasticity-regulated PI3K/AKT pathway, Proc. Natl. Acad. Sci. U.S.A. 110 (2013) 19336-19341.

[16] R. Bouchareb, M.C. Boulanger, D. Fournier, P. Pibarot, Y. Messaddeq, P. Mathieu, Mechanical strain induces the production of spheroid mineralized microparticles in the aortic valve through a RhoA/ROCK-dependent mechanism, J. Mol. Cell. Cardiol. 67 (2014) 49-59.

[17] S.T. Gould, E.E. Matherly, J.N. Smith, D.D. Heistad, K.S. Anseth, The role of valvular endothelial cell paracrine signaling and matrix elasticity on valvular interstitial, Biomaterials 35 (2014) 3596-3606.

[18] C.A. Durst, M.P. Cuchiara, E.G. Mansfield, J.L. West, K.J. Grande-Allen, Flexural characterization of cell encapsulated PEGDA hydrogels with applications for tissue engineered heart valves, Acta Biomater. 7 (2011) 2467-2476.

[19] A.M.T. Quinlan, K.L. Billiar, Investigating the role of substrate stiffness in the persistence of valvular interstitial cell activation, J. Biomed. Mater. Res. A 100A (2012) 2474-2482

[20] C.M. Kirschner, D.L. Alge, S.T. Gould, K.S. Anseth, Clickable, photodegradable hydrogels to dynamically modulate valvular interstitial cell phenotype, Adv. Healthc. Mater. 3 (2014) 649-657.

[21] A.M. Kloxin, J.A. Benton, K.S. Anseth, In situ elasticity modulation with dynamic substrates to direct cell phenotype, Biomaterials 31 (2010) 1-8. 
[22] C.Y.Y. Yip, J.H. Chen, R.G. Zhao, C.A. Simmons, Calcification by valve interstitial cells is regulated by the stiffness of the extracellular matrix, Arterioscl. Throm. Vas. 29 (2009) 936-U417.

[23] H. Tseng, M.L. Cuchiara, C.A. Durst, M.P. Cuchiara, C.J. Lin, J.L. West, K.J. Grande-Allen, Fabrication and mechanical evaluation of anatomically-inspired quasilaminate hydrogel structures with layer-specific formulations, Ann. Biomed. Eng. 41 (2013) 398-407.

[24] J. Richards, I. El-Hamamsy, S. Chen, Z. Sarang, P. Sarathchandra, M.H. Yacoub, A.H. Chester, J.T. Butcher, Side-specific endothelial-dependent regulation of aortic valve calcification interplay of hemodynamics and nitric oxide signaling, Am. J. Pathol. 182 (2013) 1922-1931.

[25] B. Duan, E. Kapetanovic, L.A. Hockaday, J.T. Butcher, Three-dimensional printed trileaflet valve conduits using biological hydrogels and human valve interstitial cells, Acta Biomater. 10 (2014) 1836-1846.

[26] P.M. Taylor, E. Sachlos, S.A. Dreger, A.H. Chester, J.T. Czernuszka, M.H. Yacoub, Interaction of human valve interstitial cells with collagen matrices manufactured using rapid prototyping, Biomaterials 27 (2006) 2733-2737.

[27] B. Duan, L.A. Hockaday, E. Kapetanovic, K.H. Kang, J.T. Butcher, Stiffness and adhesivity control aortic valve interstitial cell behavior within hyaluronic acid based hydrogels, Acta Biomater. 9 (2013) 7640-7650.

[28] Z.Y. Yin, T.M. Schmid, T.K. Yasar, Y.F. Liu, T.J. Royston, R.L. Magin, Mechanical characterization of tissue-engineered cartilage using microscopic magnetic resonance elastography, Tissue Eng. PT C-Meth. 20 (2014) 611-619.

[29] Z.Y. Yin, R.L. Magin, D. Klatt, Simultaneous MR elastography and diffusion acquisitions: Diffusion-MRE (dMRE), Magn. Reson. Med. 71 (2014) 1682-1688.

[30] C.A. Edwards, W.D. Obrien, Modified assay for determination of hydroxyproline in a tissue hydrolyzate, Clin. Chim. Acta 104 (1980) 161-167.

[31] R. Jin, L.S.M. Teixeira, P.J. Dijkstra, C.A. van Blitterswijk, M. Karperien, J. Feijen, Enzymatically-crosslinked injectable hydrogels based on biomimetic dextran-hyaluronic acid conjugates for cartilage tissue engineering, Biomaterials 31 (2010) 3103-3113. [32] R.S. Lakes. Viscoelastic Solids. Boca Raton, FL: CRC Press; 1999. 
[33] A.H. Chester, P.M. Taylor, Molecular and functional characteristics of heart-valve interstitial cells, Philos. T. Roy. Soc. B 362 (2007) 1437-1443.

[34] K.L. Sider, C.L. Zhu, A.V. Kwong, Z. Mirzaei, C.F.M. de Lange, C.A. Simmons, Evaluation of a porcine model of early aortic valve sclerosis, Cardiovasc. Pathol. 23 (2014) 289-297.

[35] K.M. Mabry, R.L. Lawrence, K.S. Anseth, Dynamic stiffening of poly(ethylene glycol)-based hydrogels to direct valvular interstitial cell phenotype in a three-dimensional environment., Biomaterials 49 (2015) 47-56.

[36] R.G. Zhao, K.L. Sider, C.A. Simmons, Measurement of layer-specific mechanical properties in multilayered biomaterials by micropipette aspiration, Acta Biomater. 7 (2011) 1220-1227.

[37] E.A. Talman, D.R. Boughner, Effect of altered hydration on the internal shear properties of porcine aortic valve cusps, Ann. Thorac. Surg. 71 (2001) S375-S378. [38] T. Meyer-ter-Vehn, H. Han, F. Grehn, G. Schlunck, Extracellular matrix elasticity modulates TGF-beta-induced p38 activation and myofibroblast transdifferentiation in human tenon fibroblasts, Invest. Ophth.Vis. Sci. 52 (2011) 9149-9155.

[39] P.J. Wipff, D.B. Rifkin, J.J. Meister, B. Hinz, Myofibroblast contraction activates latent TGF-beta 1 from the extracellular matrix, J. Cell. Biol. 179 (2007) 1311-1323. [40] B. Jian, N. Narula, Q.Y. Li, E.R. Mohler, R.J. Levy, Progression of aortic valve stenosis: TGF-beta 1 is present in calcified aortic valve cusps and promotes aortic valve interstitial cell calcification via apoptosis, Ann. Thorac. Surg. 75 (2003) 457-465. [41] N. Huebsch, P.R. Arany, A.S. Mao, D. Shvartsman, O.A. Ali, S.A. Bencherif, J. Rivera-Feliciano, D.J. Mooney, Harnessing traction-mediated manipulation of the cell/matrix interface to control stem-cell fate, Nat. Mater. 9 (2010) 518-526.

[42] K. Chatterjee, S. Lin-Gibson, W.E. Wallace, S.H. Parekh, Y.J. Lee, M.T. Cicerone, M.F. Young, C.G. Simon, The effect of 3D hydrogel scaffold modulus on osteoblast differentiation and mineralization revealed by combinatorial screening, Biomaterials 31 (2010) 5051-5062.

[43] S.H. Parekh, K. Chatterjee, S. Lin-Gibson, N.M. Moore, M.T. Cicerone, M.F. Young, C.G. Simon, Modulus-driven differentiation of marrow stromal cells in 3D scaffolds that is independent of myosin-based cytoskeletal tension, Biomaterials 32 (2011) 2256-2264. 
[44] R. McBeath, D.M. Pirone, C.M. Nelson, K. Bhadriraju, C.S. Chen, Cell shape, cytoskeletal tension, and RhoA regulate stem cell lineage commitment, Dev. Cell 6 (2004) 483-495.

[45] K.A. Kilian, M. Mrksich, Directing stem cell fate by controlling the affinity and density of ligand-receptor interactions at the biomaterials interface, Angew. Chem. Int. Edit. 51 (2012) 4891-4895.

[46] S. Khetan, J.A. Burdick, Patterning network structure to spatially control cellular remodeling and stem cell fate within 3-dimensional hydrogels, Biomaterials 31 (2010) 8228-8234.

[47] X.X. Gu, K.S. Masters, Role of the Rho pathway in regulating valvular interstitial cell phenotype and nodule formation, Am. J. Phsiol.-Heart C. 300 (2011) H448-H458. [48] X.X. Gu, K.S. Masters, Role of the MAPK/ERK pathway in valvular interstitial cell calcification, Am. J. Phsiol.-Heart C. 296 (2009) H1748-H1757.

[49] C.M. Alfieri, J. Cheek, S. Chakraborty, K.E. Yutzey, Wnt signaling in heart valve development and osteogenic gene induction, Dev. Biol. 338 (2010) 127-135. 


\section{List of Figures}

Fig. 1 Schematic illustration of HAVIC-laden hydrogels fabrication. (A) Homogeneous hydrogels: cells and hydrogel precursors were loaded into silicone molds and subsequently exposed to $365 \mathrm{~nm} \mathrm{UV} \mathrm{light} \mathrm{for} 1 \mathrm{~min}$ or $5 \mathrm{~min}$. The cell-hydrogel constructs were maintained in control medium (CTL) for 1 day and then cultured in CTL, activation medium (TGF $\beta 1$ ), or osteogenic medium (OGM) for another 20 days; (B) heterogeneous hydrogels: Heterogeneous photocrosslinking of cell laden hydrogels were fabricated by exposing the hydrogels to UV light for different time in the same construct. The cell-hydrogel precursor solution was loaded in a square silicone mold and photocrosslinked for $1 \mathrm{~min}$. Then a totally black photomask was used to cover part of the hydrogels to block the UV light and then the constructs were further exposed to UV light for another $4 \mathrm{~min}$. Part of the construct was photocrosslinked for $1 \mathrm{~min}$ and the other part was photocrosslinked for totally $5 \mathrm{~min}$; (C) in situ stiffening HAVIC laden hydrogels: the constructs with 1 min photocrosslink were cultured in OGM for 7 days and then were stiffened via radical polymerisation by UV exposure for another $4 \mathrm{~min}$ after incubation in a 6-well plate with Irgacure 2959 containing media (0.05 wt\%) for $30 \mathrm{~min}$ at $37^{\circ} \mathrm{C}$. Immediately after exposure, the constructs were washed three times with fresh medium and conditioned in OGM for another 14 days.

Fig. 2 (A-C) Tunable mechanical properties of accellular hydrogels by varying photocrosslink time. (A) Uniaxial compressive tests were conducted and compressive moduli were calculated using a dynamic mechanical analyzer (DMA). With increasing the photocrosslink time from $1 \mathrm{~min}$ to $5 \mathrm{~min}$, the 
stiffness of hybrid hydrogels significantly increased from $1.33 \pm 0.17 \mathrm{kPa}$ to $3.33 \pm 0.19 \mathrm{kPa}$. We use the initial stiffness of $1 \mathrm{kPa}$ and $3 \mathrm{kPa}$ to represent the 1 min and 5 min photocrosslink hydrogel constructs. (B, C) Shear stiffness measured by microscopic magnetic resonance elastography ( $\mu$ MRE). (B) Shear wave images obtained from phase part of the complex MR images (left) and shear stiffness maps obtained using the local frequency estimation (right); (C) shear stiffness calculated based on the propagating wavelength and wave images. (** $p<0.01 ; \mathrm{n}=6$ for DMA test; $\mathrm{n}=3$ for $\mu \mathrm{MRE}$ test). (D, E) Dynamic changes of compressive stiffness of HAVIC populated hydrogels with different initial accellular stiffness. (D) HAVIC laden hydrogels with 5 min photocrosslink time and $3 \mathrm{kPa}$ initial accellular stiffness; (E) HAVIC laden hydrogels with $1 \mathrm{~min}$ photocrosslink time and $1 \mathrm{kPa}$ initial accellular stiffness (dash line indicated the compressive stiffness of accellular hydrogels; $\mathrm{n}=6$; ${ }^{* *} p<0.01$; bars that do not share letters are significantly different from each other; $\$$ indicated no significantly difference between 7-day in OGM and 21-day in TGF $\beta 1$ ).

Fig. 3 Dynamic changes of ECM contents. Quantitative analysis of GAGs (A), collagen (B) and elastin (C) content for HAVIC-hydrogel constructs as a function of time. $(n=5-6$; bars that do not share letters are significantly different from each other; $\$$ indicated $\mathrm{p}>0.05$ comparing to $1 \mathrm{kPa}$ constructs conditioned in activation media at day 21; \# indicated $\mathrm{p}<0.05$ comparing to $3 \mathrm{kPa}$ hydrogels in control media at day 21); (D) dynamic changes of total GAG, collagen and elastin content with stiffness under different conditions from day 7 to day 21 (Red lines present changes of $1 \mathrm{kPa}$ hydrogels from day 7 to day 21; blue lines present changes of 3 
kPa hydrogels; triangle presents control media; square presents activation media; circle presents osteogenic media; arrow indicates the changes from day 7 to day $21 ;{ }^{*} p<0.05, * * p<0.01$ indicate significant difference for compressive stiffness between day 7 and day 21).

Fig. 4 Hydrogel stiffness and culture conditions dynamically affect myofibroblast-like and osteogenic-like phenotypes of HAVIC. Representative immunohistochemical staining images of (A) vimentin (green), alpha smooth muscle actin ( $\alpha$ SMA, red) and (B) Runx2 (green); osteocalcin (OCN, green); (nuclei: blue; scale bar: $20 \mu \mathrm{m})$; (C) dynamic changes of gene expression (vimentin, $\alpha \mathrm{SMA}$, Runx2 and OCN) with stiffness under different conditions from day 7 to day 21. (Red lines present changes of $1 \mathrm{kPa}$ hydrogels; blue lines present changes of $3 \mathrm{kPa}$ hydrogels; triangle presents control media; square presents activation media; circle presents osteogenic media; arrow indicates the changes from day 7 to day $21 ;{ }^{*} p<0.05$, ${ }^{* *} p<0.01$ indicate significant difference for gene expression between day 7 and day 21; relative gene expression is presented as normalized to $18 \mathrm{~s}$ and expressed relative to $1 \mathrm{kPa}$ hydrogel in control media).

Fig. 5 Dynamic changes of ALP expression and activity with stiffness under different conditions from day 7 to day 21. (A) Representative ALP staining images (scale bar: $400 \mu \mathrm{m}$ ); (B) hydrogel with lower initial stiffness and osteogenic media promote ALP activity. (Red lines present changes of $1 \mathrm{kPa}$ hydrogels; blue lines present changes of $3 \mathrm{kPa}$ hydrogels; triangle presents control media; square presents activation media; circle presents osteogenic media; arrow indicates the 
changes from day 7 to day $21 ; * * p<0.01$ indicate significant difference for ALP activity between day 7 and day 21).

Fig. 6 Heterogeneous hydrogel constructs confirm that $1 \mathrm{kPa}$ hydrogels express more ALP. (A) ALP staining image of heterogeneous hydrogel construct generated by exposing the HAVIC-laden hydrogels to UV light for different time. The dash line indicates the boundary between $1 \mathrm{kPa}$ and $3 \mathrm{kPa}$ hydrogel; (B) qPCR result shows that HAVIC in $1 \mathrm{kPa}$ hydrogels significantly upregulate ALP expression $(* * p<0.01$; relative gene expression is presented as normalized to $18 \mathrm{~s}$ and expressed relative to $3 \mathrm{kPa}$ hydrogel in osteogenic media).

Fig. 7 In situ temporal stiffening regulates HAVIC osteogenic differentiation. (A) Compaction ratio of HAVIC laden hydrogels with different initial stiffness and in situ stiffening (upper images are representative hydrogel images at day 21); (B) compressive stiffness of $1 \mathrm{kPa}, 3 \mathrm{kPa}$ and in situ-stiffened hydrogels at day 7 and 21 (day 7 indicates the time point for in situ-stiffening, and day 21 indicates 14 days after in situ-stiffening); temporal stiffening process decreases ALP activity (C) and downregulated gene expressions of ALP and OCN (D) compared to $1 \mathrm{kPa}$ hydrogels at day 21 in OGM $\left({ }^{*} p<0.05,{ }^{*} p<0.01\right.$; relative gene expression is presented as normalized to $18 \mathrm{~s}$ and expressed relative to $1 \mathrm{kPa}$ hydrogel in OGM).

Fig. 8 Effects of ROCK inhibitor and activator on HAVIV phenotypes. (A) Gene expressions of vimentin, $\alpha$ SMA, Runx2 and OCN in HAVIC within $1 \mathrm{kPa}$ hydrogels treated with ROCK inhibitor (Y27632, $10 \mu \mathrm{M}$ ) or activator (LPA, 20 $\mu \mathrm{M})$ (relative gene expression is presented as normalized to $18 \mathrm{~S}$ and expressed relative to $1 \mathrm{kPa}$ hydrogel in $\mathrm{OGM}$ ); (B) western blot bands and quantification of 
total RhoA and OCN expression normalized to GAPDH ratio after 21 days in osteogenic media. (Bars that do not share letters are significantly different from each other)

Fig. 9 Proposed schematic diagram of CAVD progression and mechanical and biochemical regulation of VIC phenotypes. (A) It is elusive how ECM mechanical properties change from compliant healthy valve to stiff calcified valve. There may have several potential mechanisms whereby matrix stiffness has different change rates and forms. (I) Matrix stiffness increases at approximately constant rate; (II) matrix stiffness slowly increases and then rapidly increases; (III) matrix stiffness decreases at the early stage and then increases; (a-d) indicate the microenvironments at different stages; (B) model of mechanical and biochemical regulation of VIC phenotypes. Within pathological microenvironment(s), the constructs with stiffness lower than normal level facilitate osteogenic differentiation of HAVIC and the differentiation is mediated by RhoA/ROCK signaling pathway. Temporal stiffening of lower stiffness matrix to valve leaflet layers-like stiffness or blocking RhoA/ROCK signaling pathway activates HAVIC and may delay or partially inhibit osteogenic differentiation process. 
Fig. 1

A

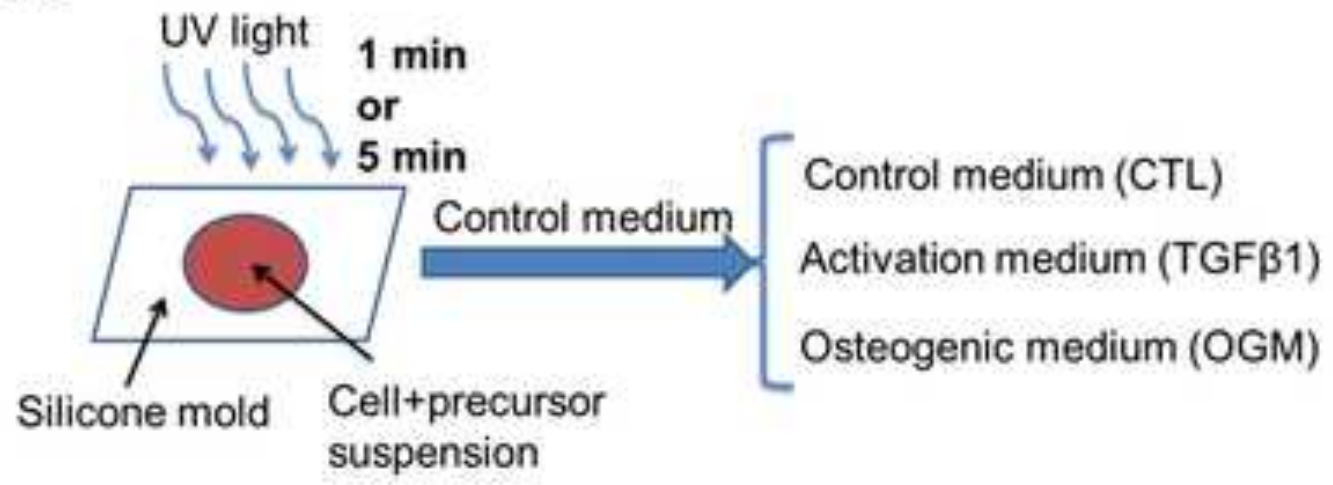

B
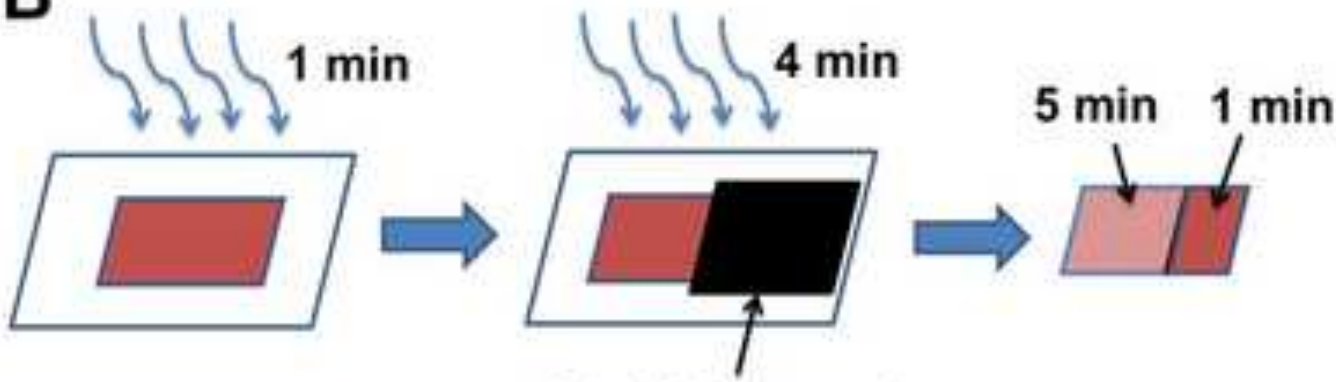

Black Photomask

C

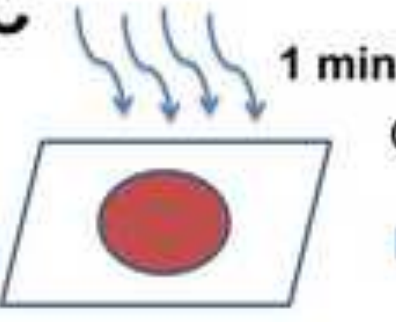

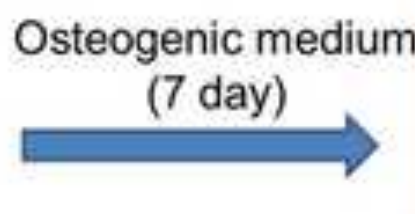

Incubate in control medium with Irgacure $0.05 \%$ for $30 \mathrm{~min}$

Osteogenic medium ( 14 day)
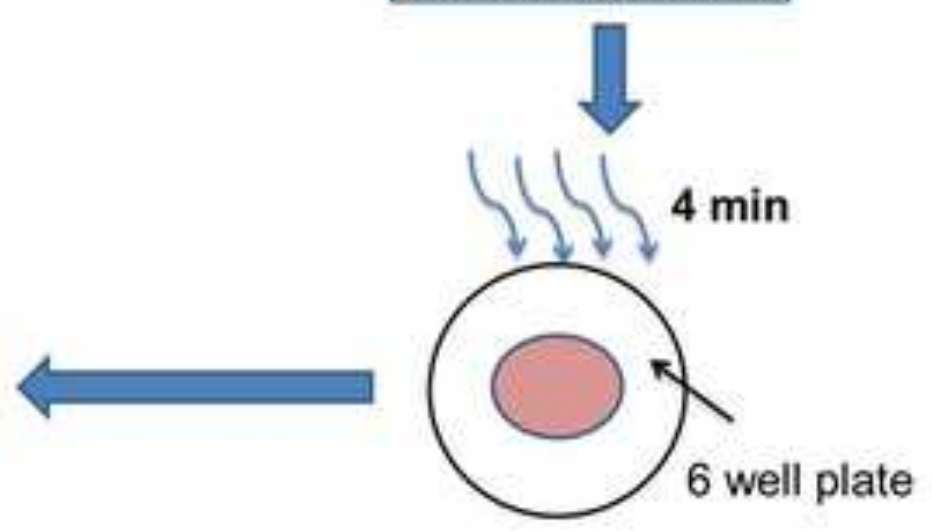
Fig. 2
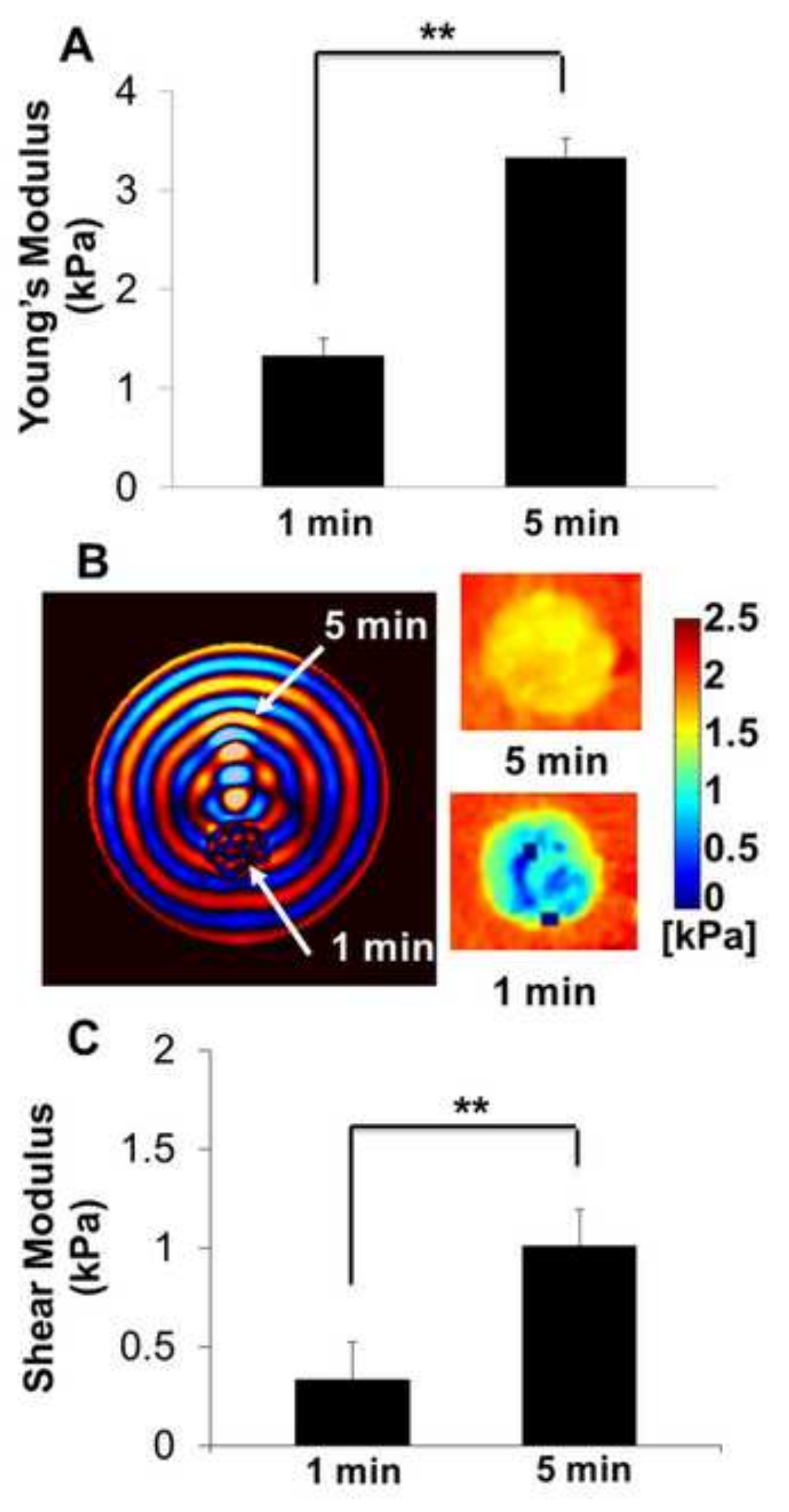$$
\text { . }
$$
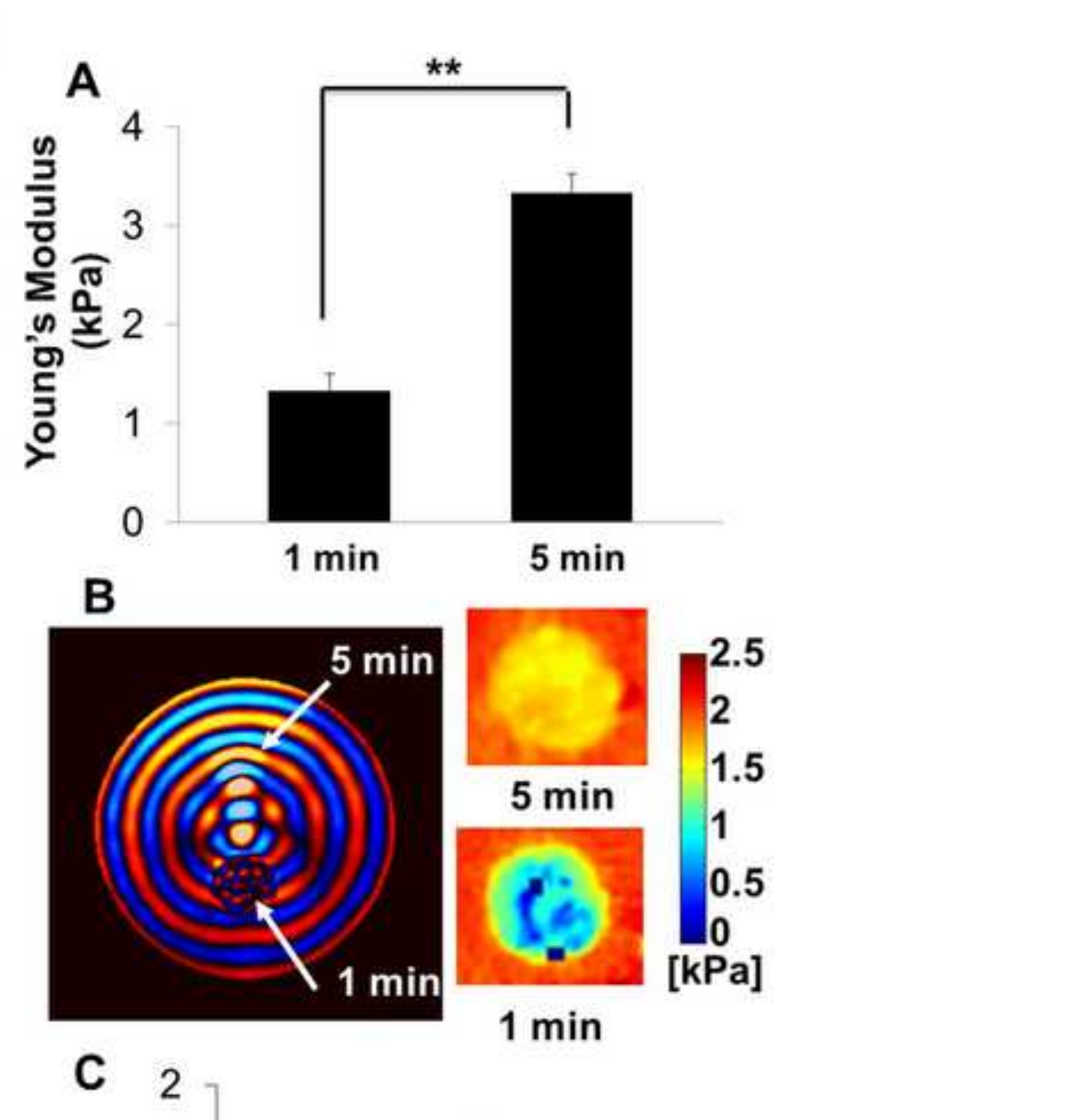

$+2$

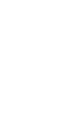

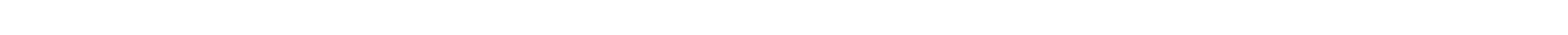


Fig. 2
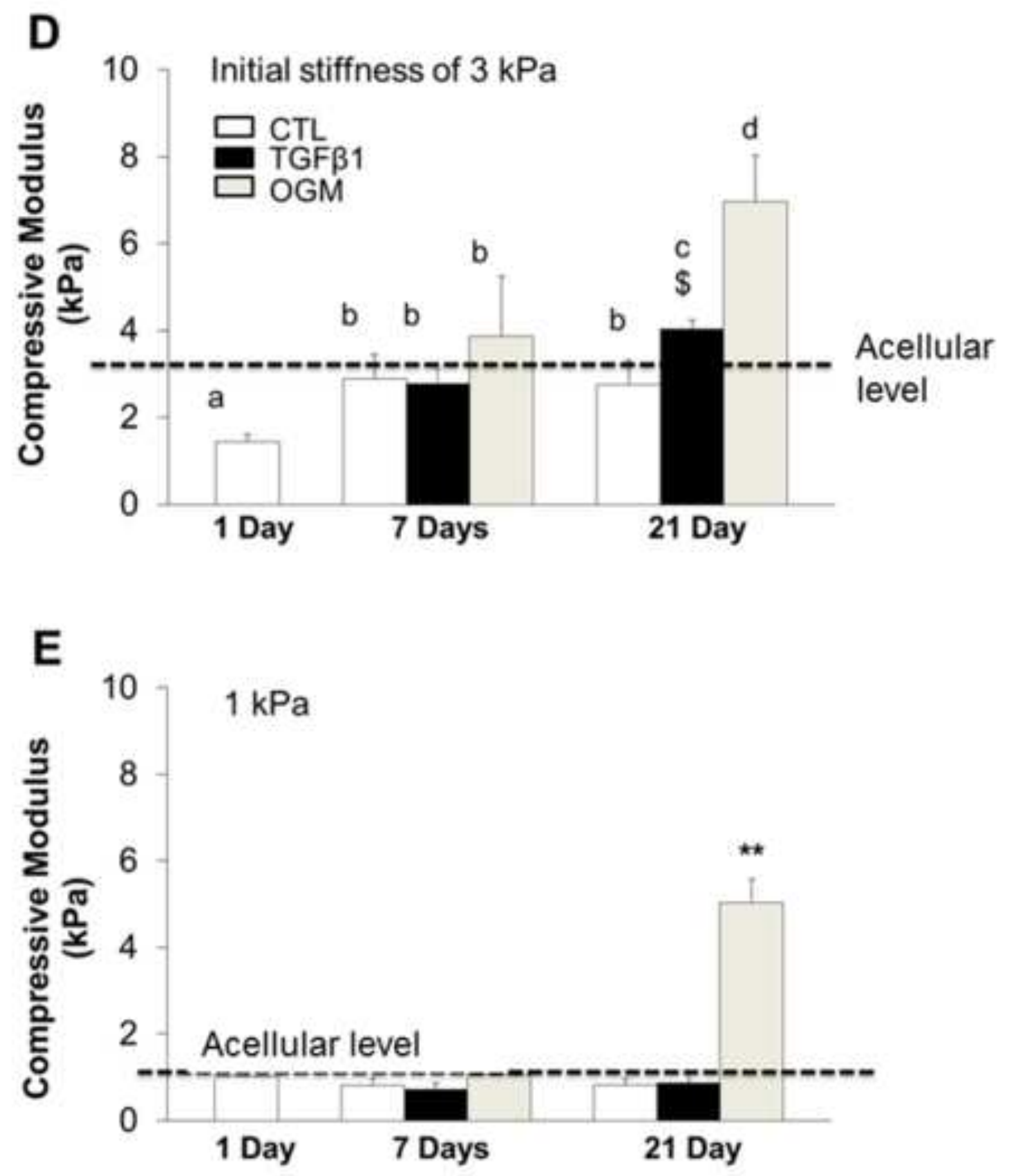

Figure 2D-E 
Fig. 3
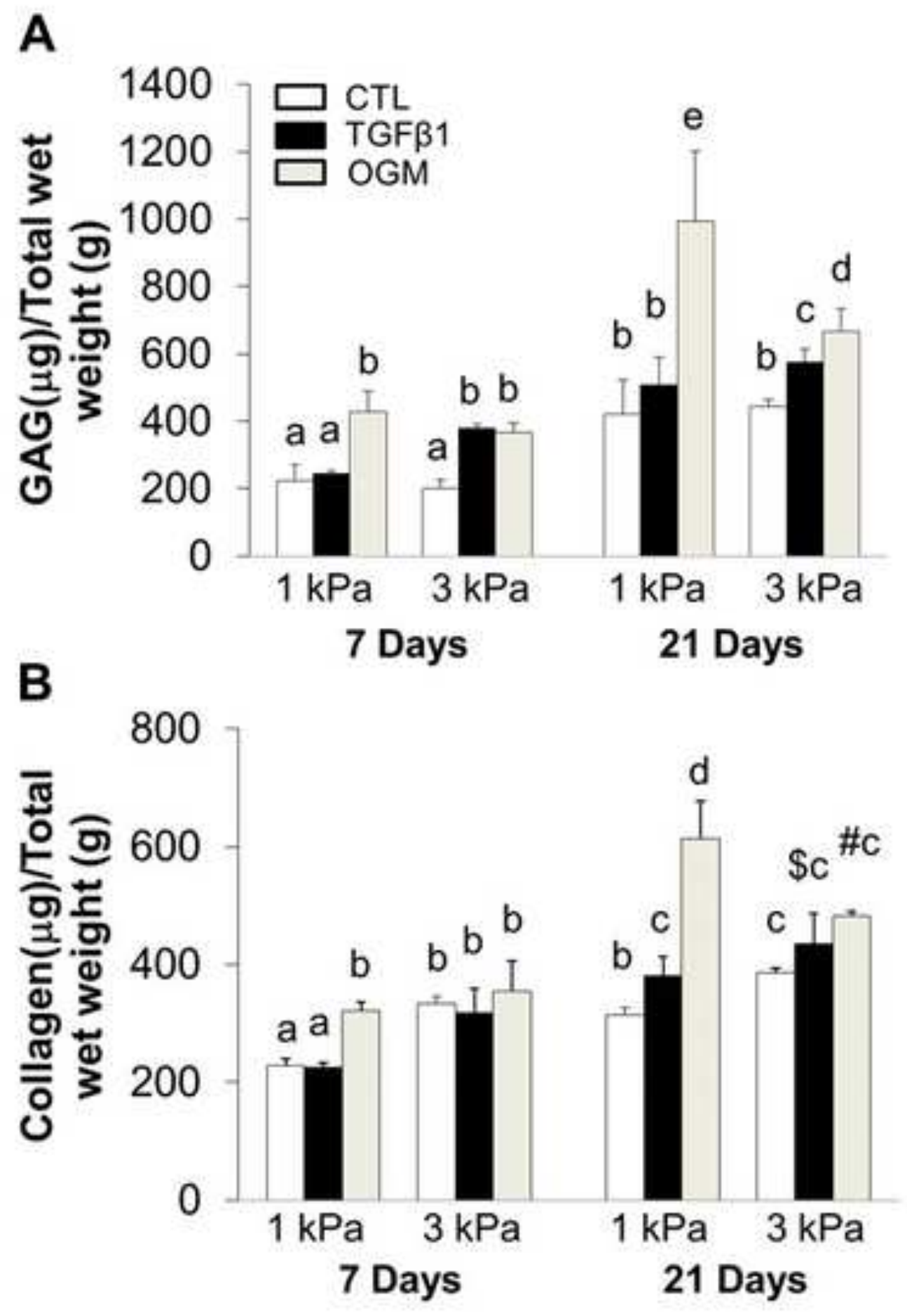
Fig. 3
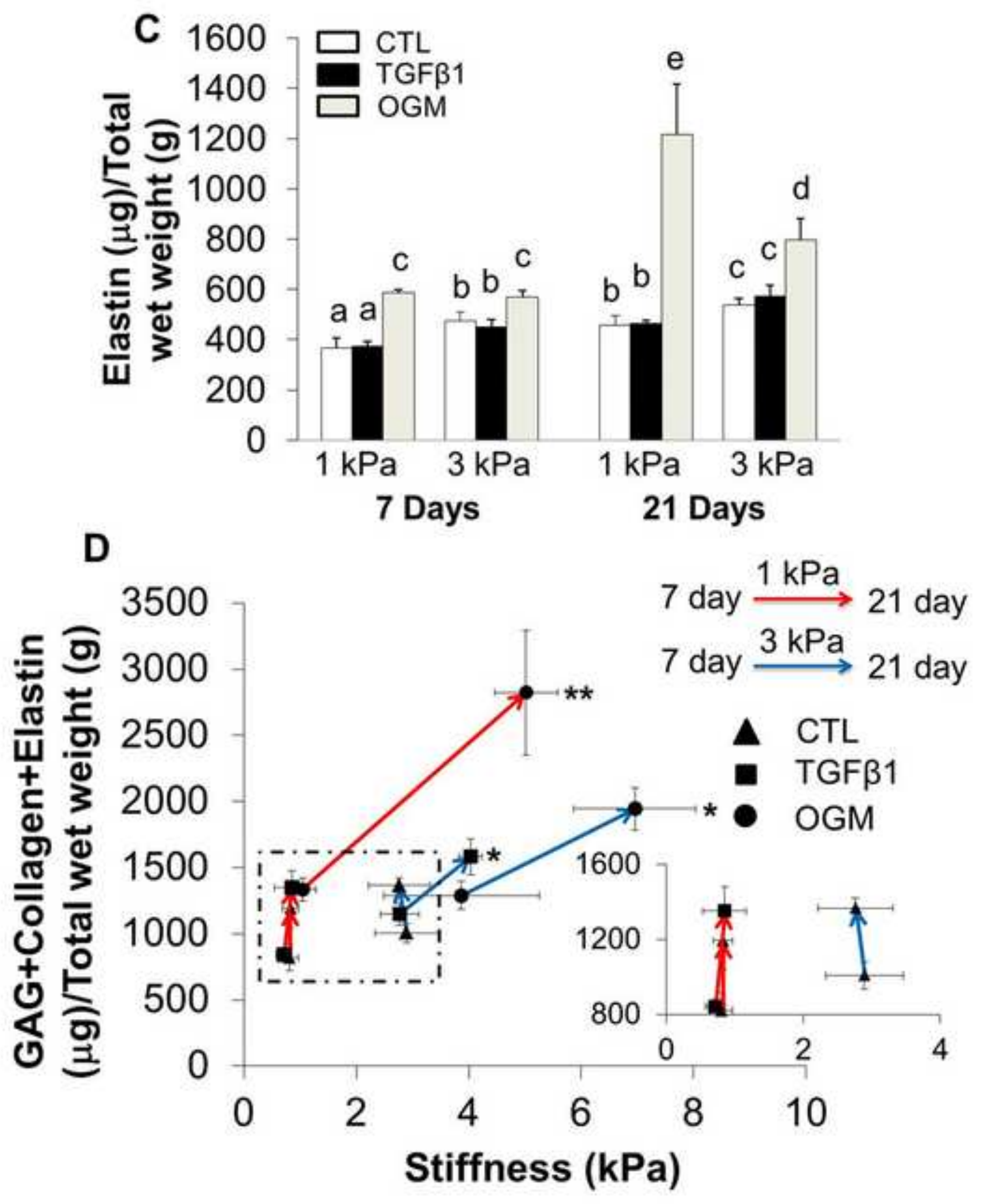

Figure 3C-D 
Fig. 4
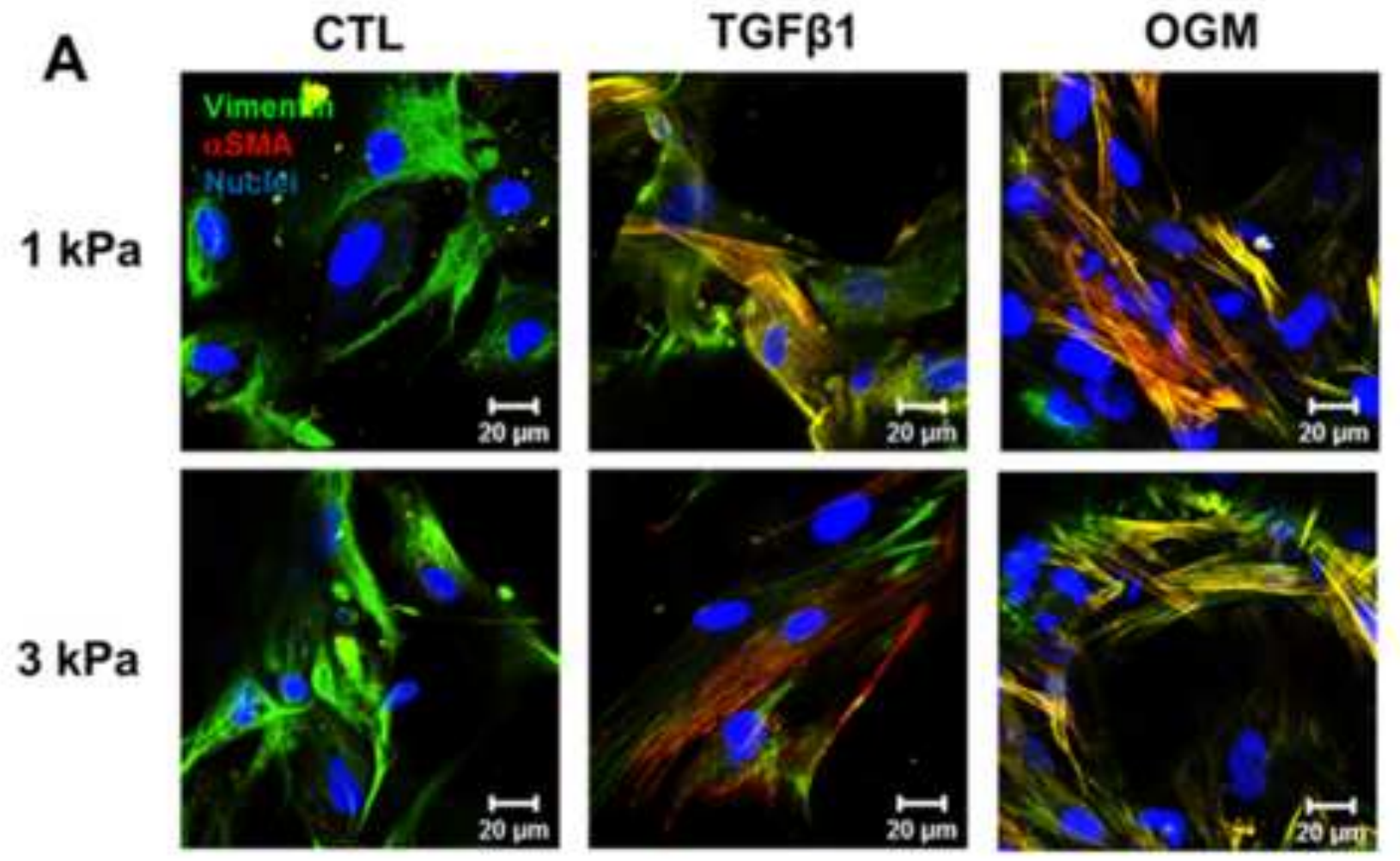

Figure 4A

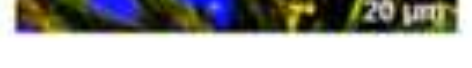

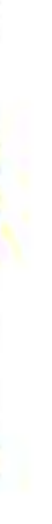

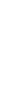


Fig. 4
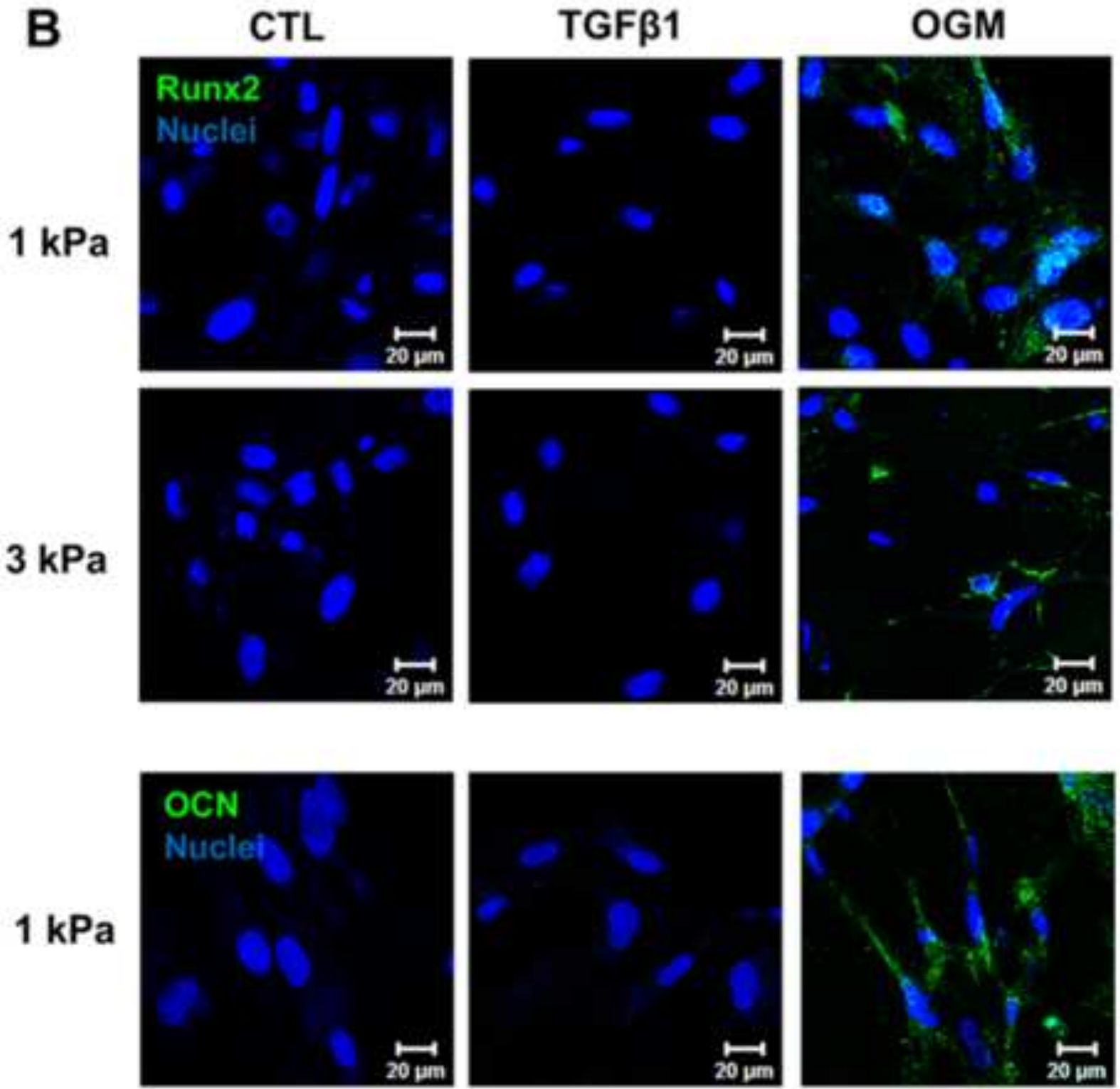

$3 \mathrm{kPa}$
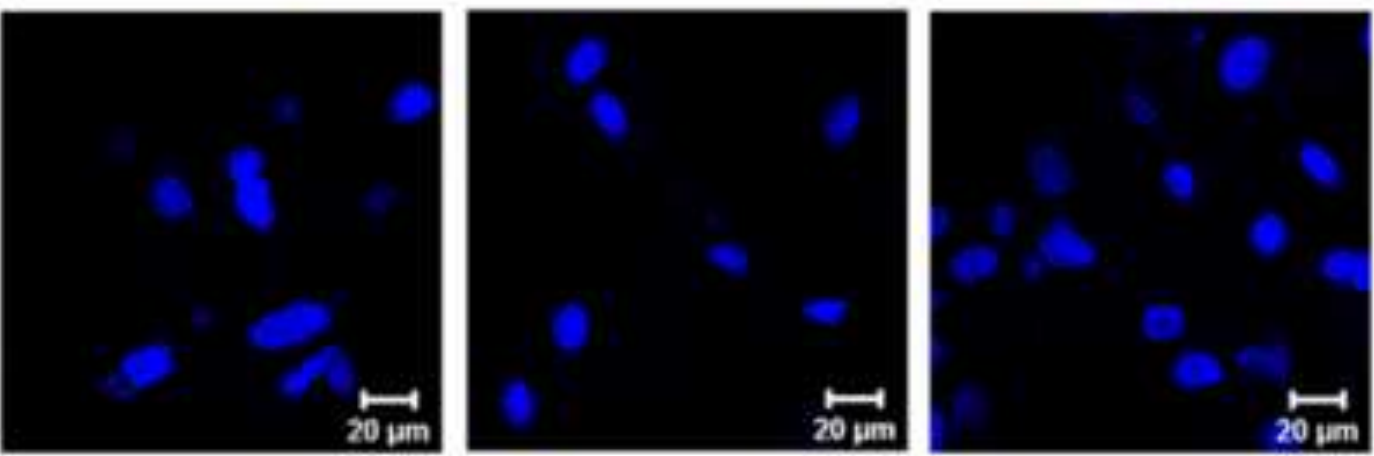

$3 \mathrm{kPa}$ 
Fig. 4
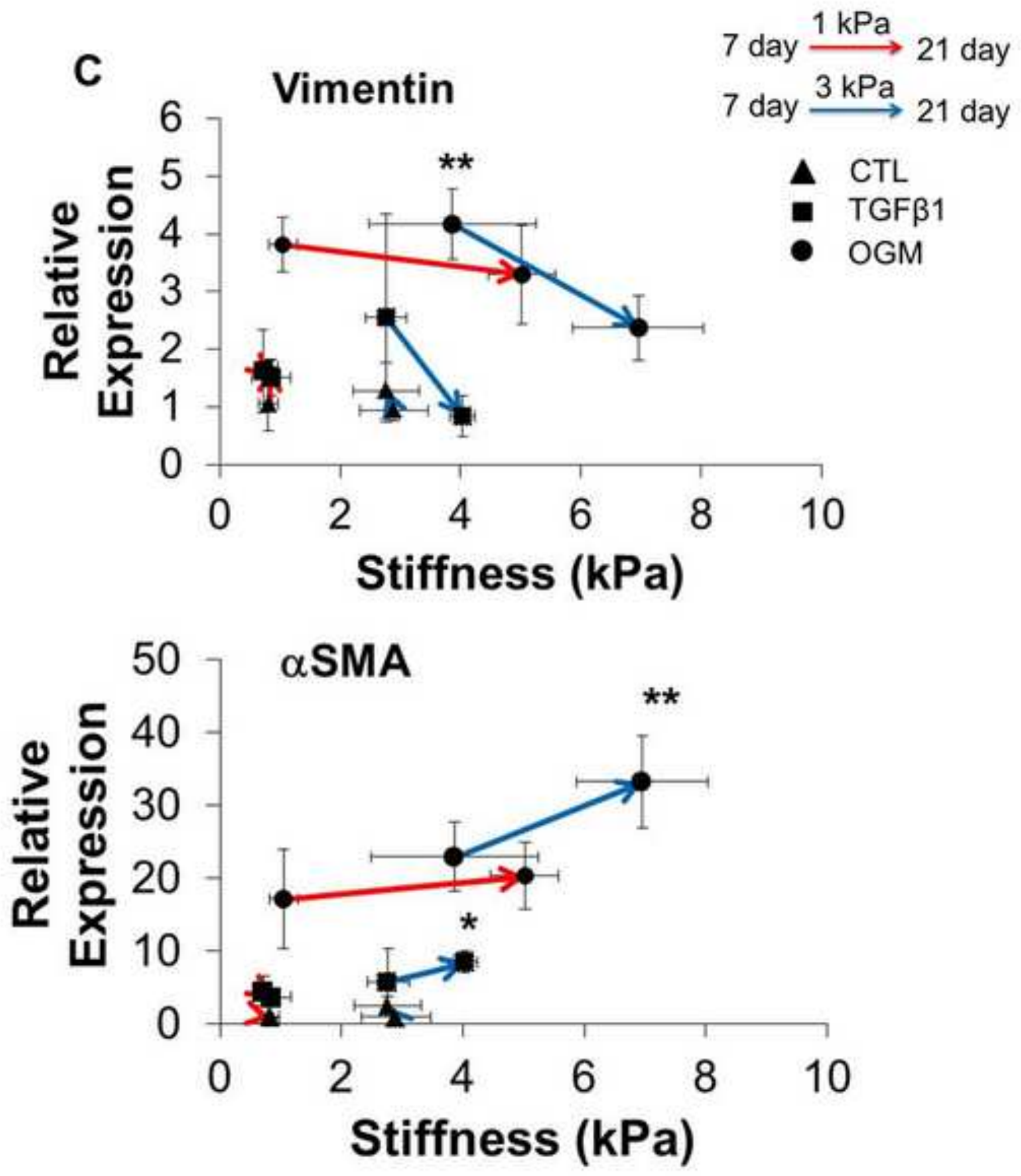

Figure $4 \mathrm{C} 1$

C

Vimentin

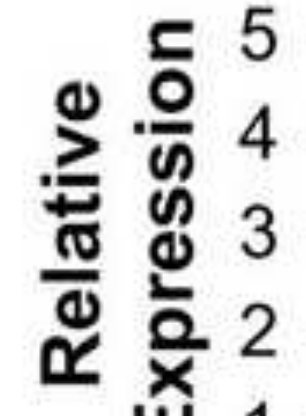

0 
Fig. 4
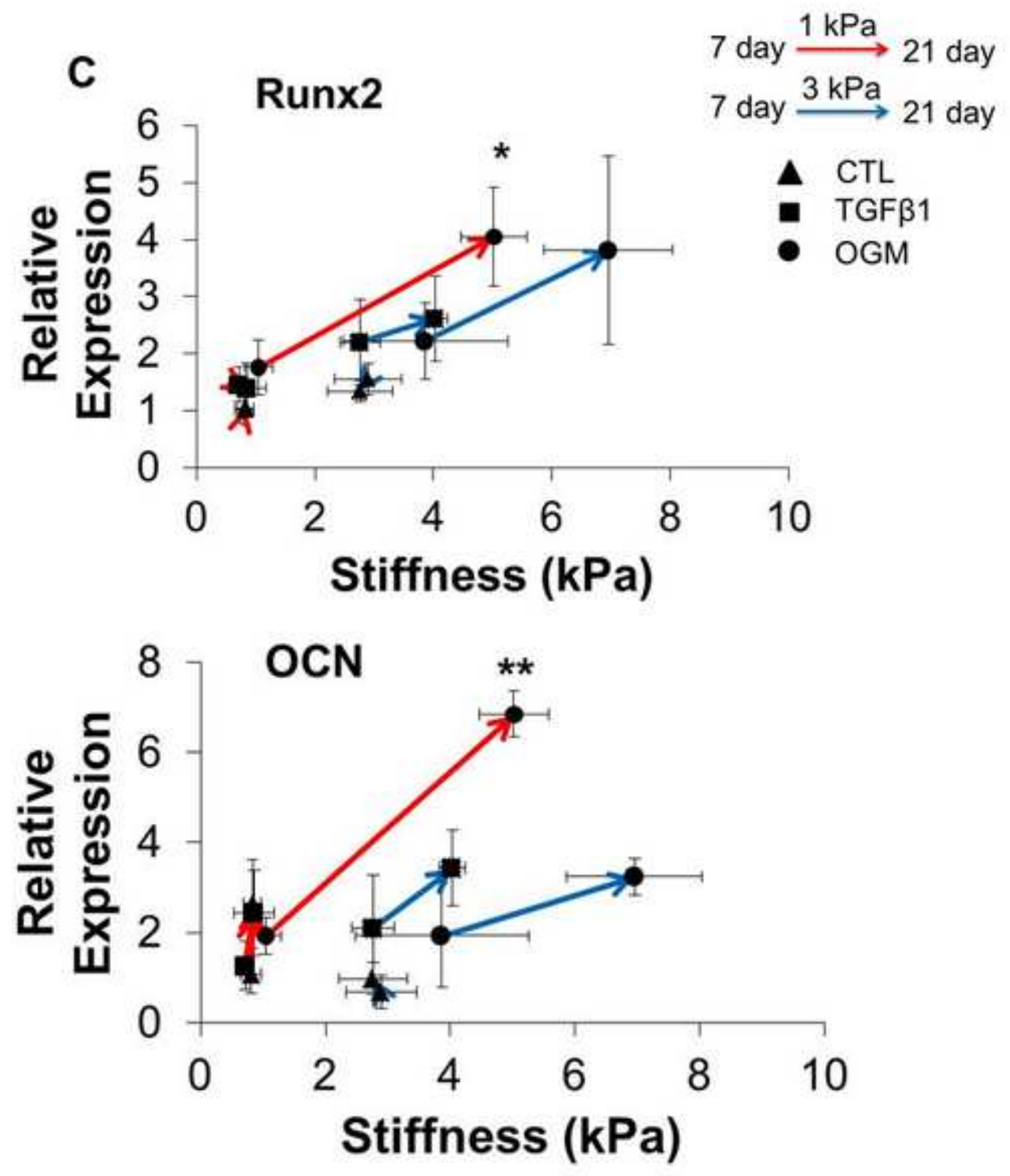
Fig. 5
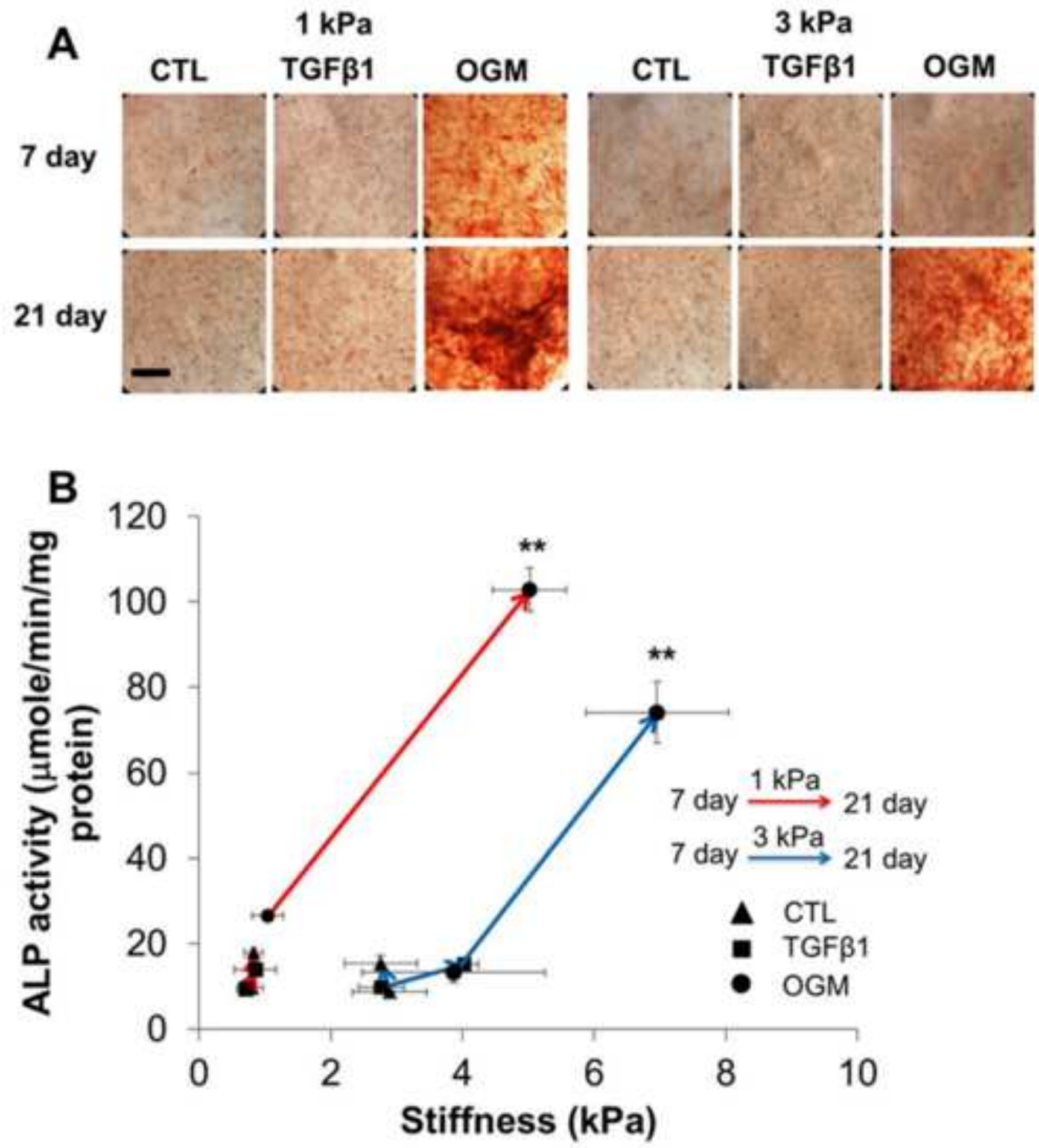
Fig. 6
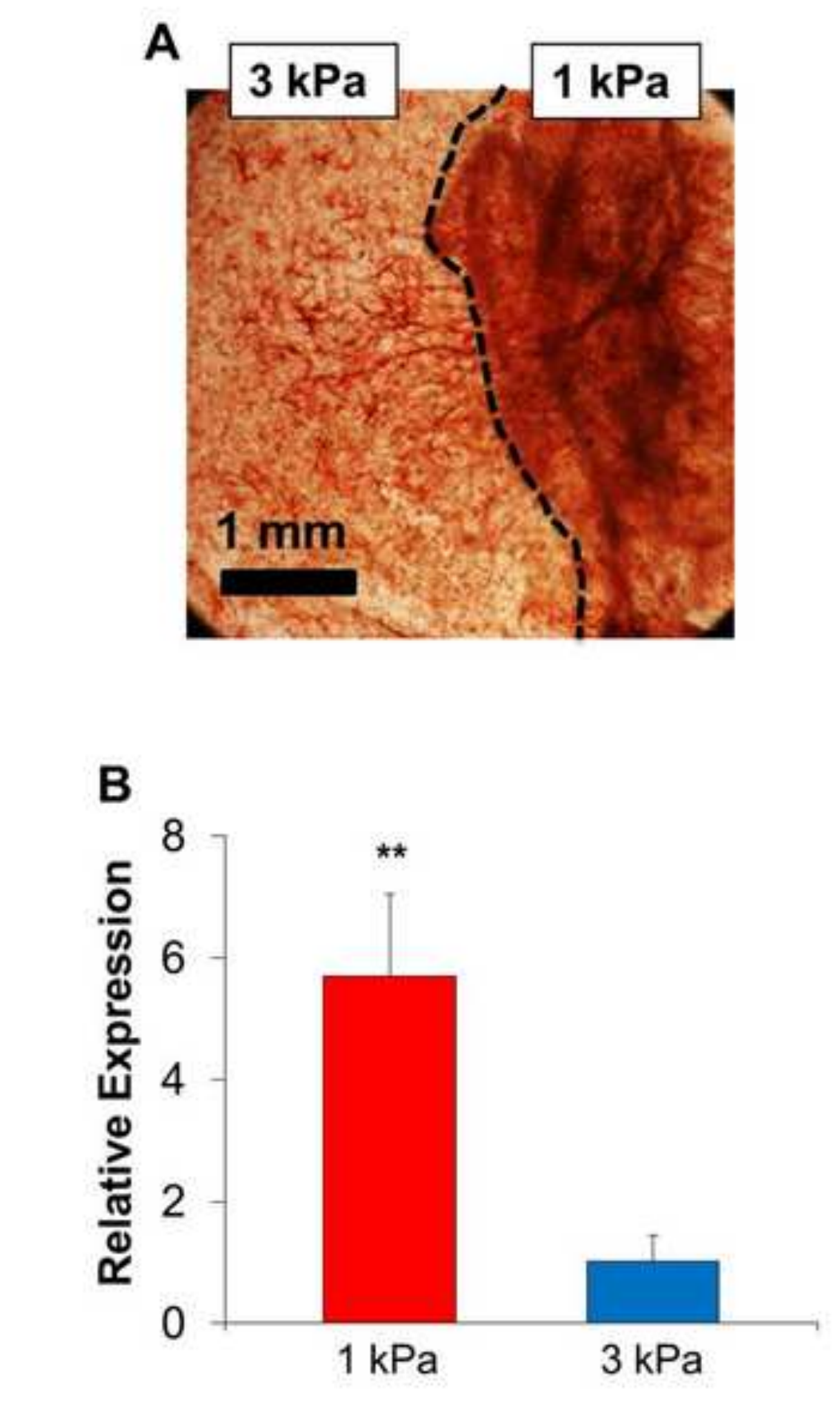

Figure 6
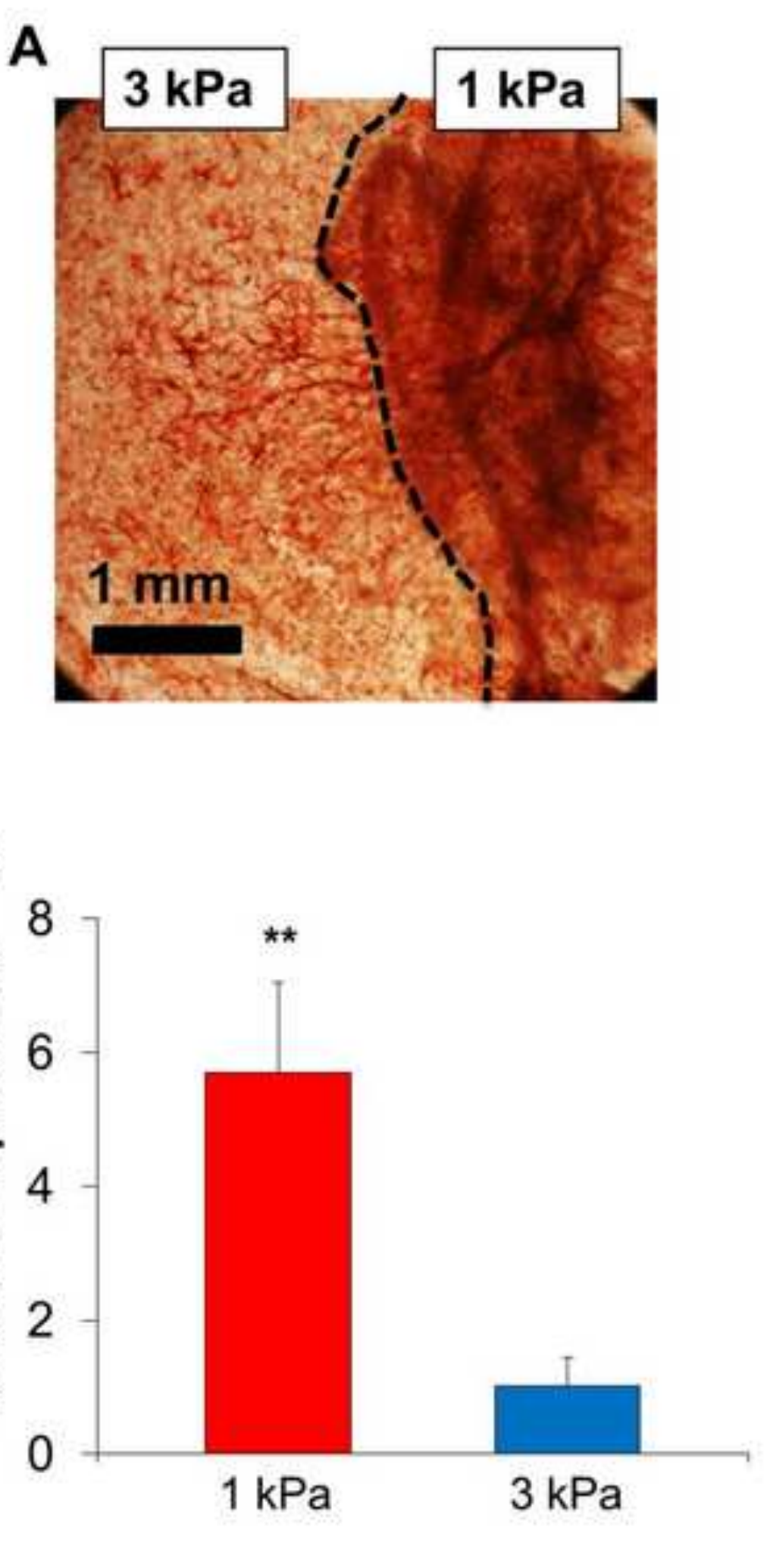

$$
\text { . }
$$


Fig. 7
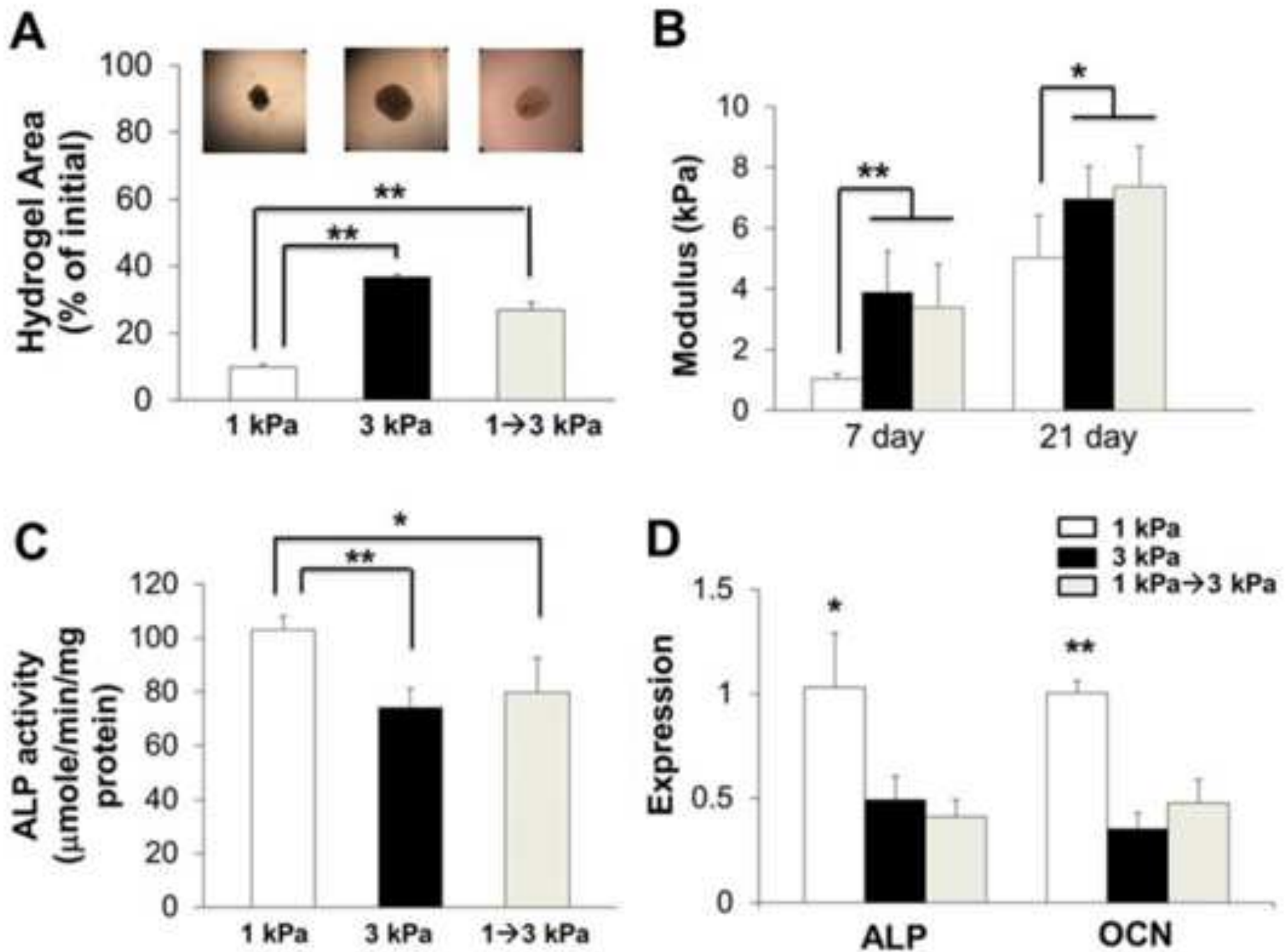
Fig. 8
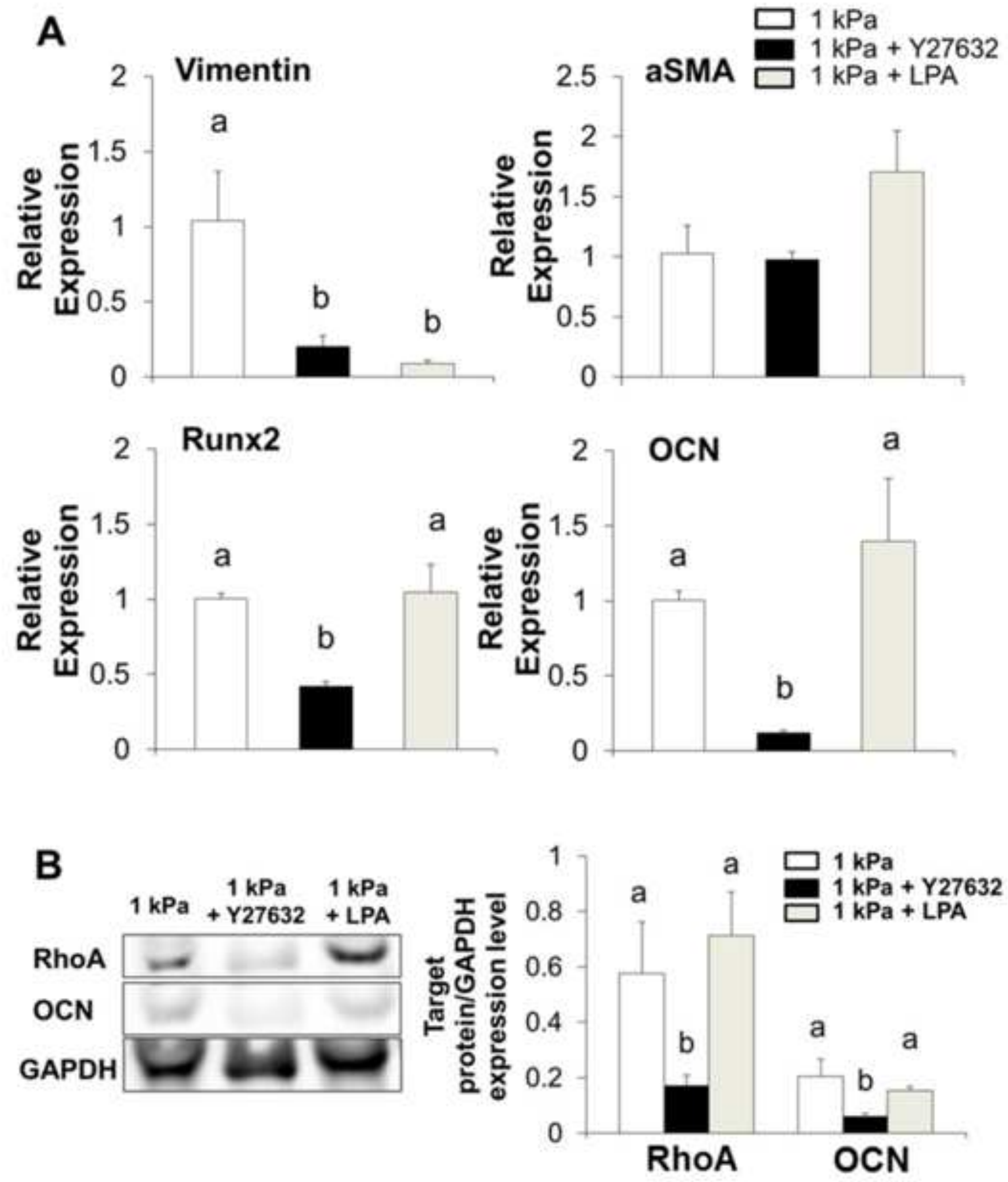
Fig. 9
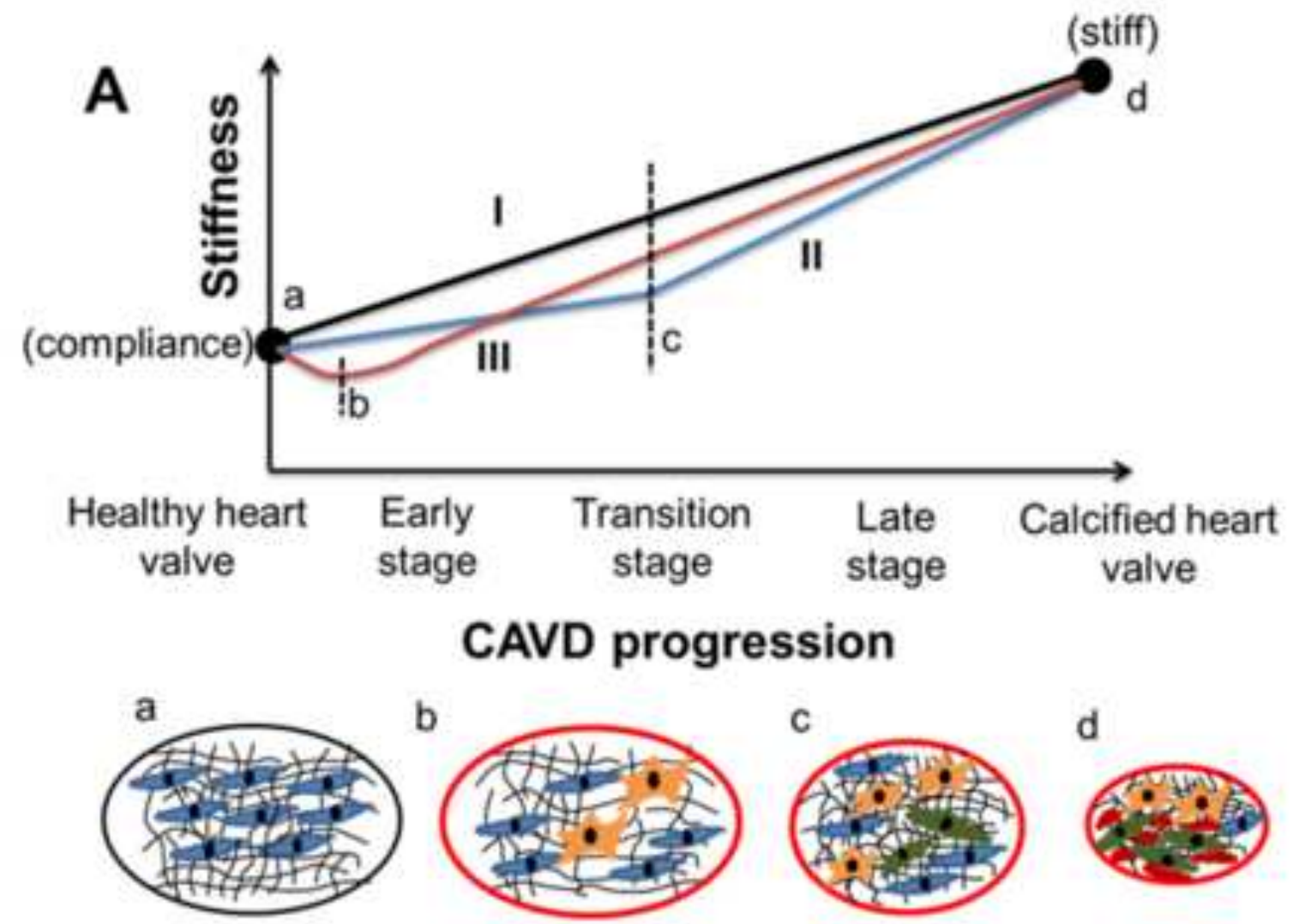

B

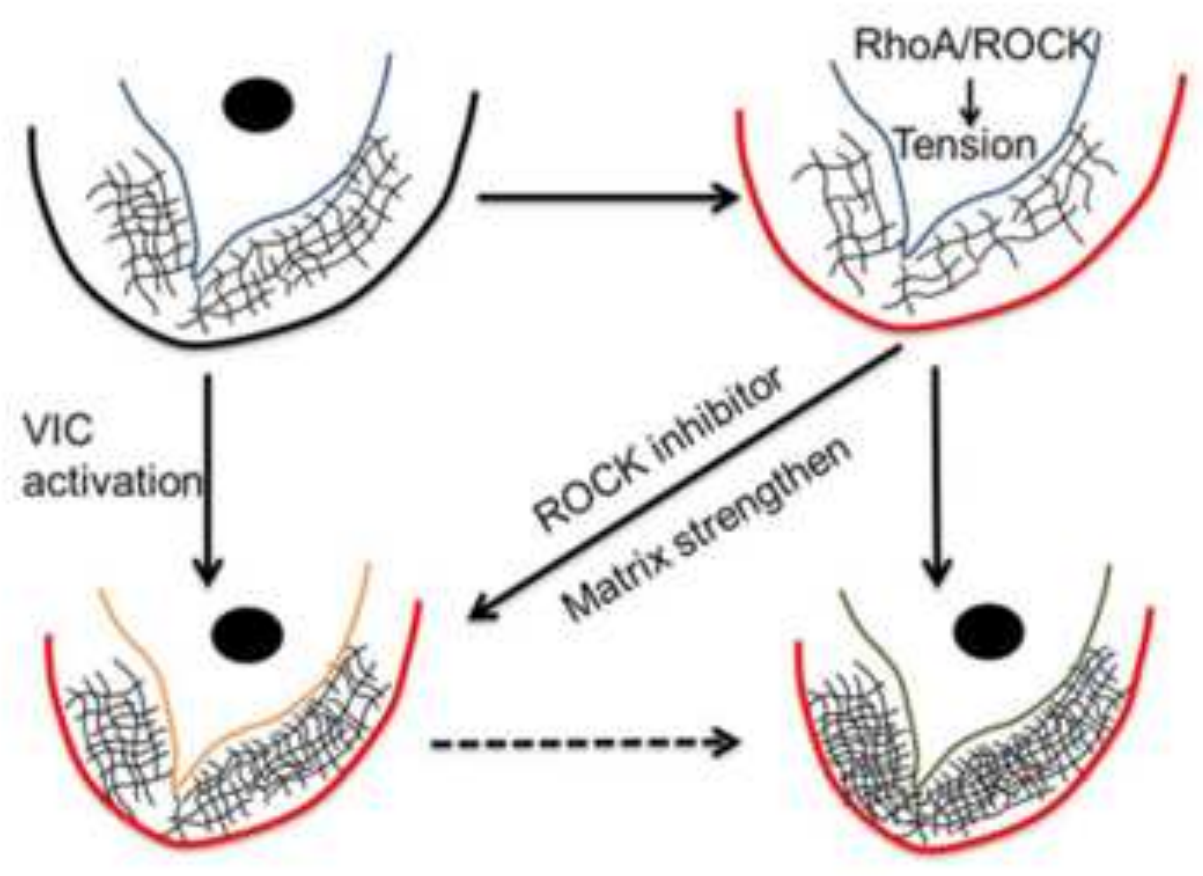
Physiological microenvironment
Pathological microenvironment
- Quiescent VIC Osteoblast-like VIC
- Calcium accumulation - Myofibroblast-like VIC

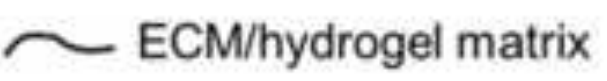




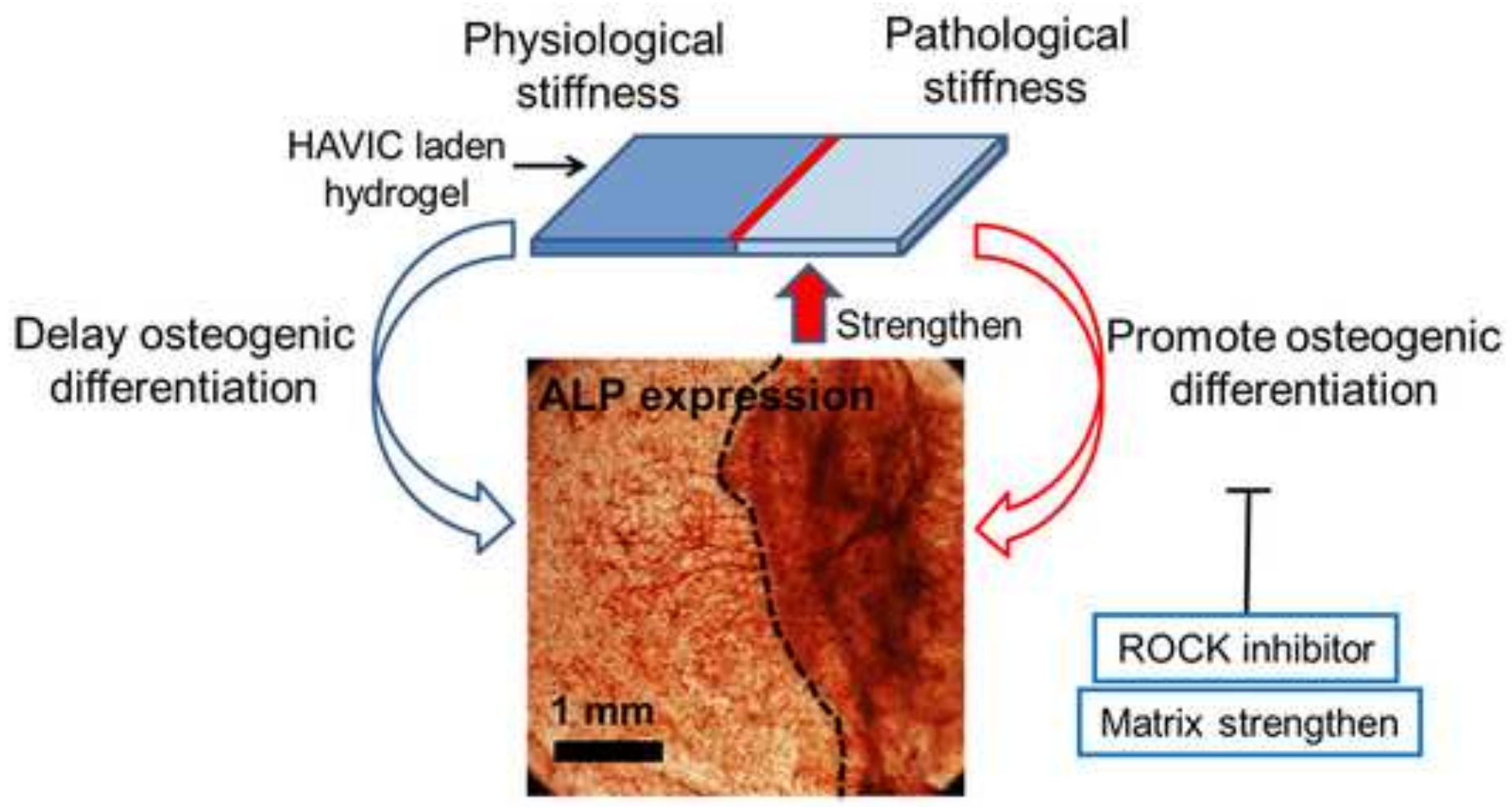

